QUARTERLY OF APPLIED MATHEMATICS

VOLUME LXV, NUMBER 1

MARCH 2007, PAGES 163-187

S 0033-569X(07)01047-4

Article electronically published on February 12, 2007

\title{
MILD SOLUTIONS FOR THE RELATIVISTIC VLASOV-MAXWELL SYSTEM FOR LASER-PLASMA INTERACTION
}

\author{
BY \\ MIHAI BOSTAN \\ Laboratoire de Mathématiques de Besançon, Université de Franche-Comté, 16 route de Gray 25030 \\ Besançon France
}

\begin{abstract}
We study a reduced 1D Vlasov-Maxwell system which describes the laserplasma interaction. The unknowns of this system are the distribution function of charged particles, satisfying a Vlasov equation, the electrostatic field, verifying a Poisson equation and a vector potential term, solving a nonlinear wave equation. The nonlinearity in the wave equation is due to the coupling with the Vlasov equation through the charge density. We prove here the existence and uniqueness of the mild solution (i.e., solution by characteristics) in the relativistic case by using the iteration method.
\end{abstract}

1. Introduction. We consider a population of relativistic electrons with mass $m>0$ and charge $-e<0$. We denote by $v(p)=\frac{p}{m}\left(1+\frac{|p|^{2}}{m^{2} c^{2}}\right)^{-1 / 2}$ the velocity associated to a given momentum $p \in \mathbb{R}^{3}$, where $c$ is the light speed. The electrons move under the action of an electric field $E$ and a magnetic field $B$. Their distribution function $F=F(t, x, p)$ satisfies the Vlasov equation

$$
\left.\partial_{t} F+v(p) \cdot \nabla_{x} F-e(E(t, x)+v(p) \wedge B(t, x)) \cdot \nabla_{p} F=0, \quad(t, x, p) \in\right] 0, T\left[\times \mathbb{R}^{3} \times \mathbb{R}^{3} .\right.
$$

The electro-magnetic field verifies the Maxwell equations

$$
\partial_{t} E-c^{2} \operatorname{curl} B=\frac{e}{\varepsilon_{0}} j, \partial_{t} B+\operatorname{curl} E=0, \operatorname{div} E=\frac{e}{\varepsilon_{0}}\left(\rho_{\text {ext }}-\rho\right), \operatorname{div} B=0,
$$

where $\varepsilon_{0}$ is the dielectric permittivity of vacuum, $\rho_{\text {ext }}$ is the density of a background population of ions which are supposed at rest and the electron density $\rho$ and current $j$ are given by

$$
\rho(t, x)=\int_{\mathbb{R}^{3}} F(t, x, p) d p, \quad j(t, x)=\int_{\mathbb{R}^{3}} v(p) F(t, x, p) d p, \quad \forall(t, x) \in[0, T] \times \mathbb{R}^{3} .
$$

The Cauchy problem of the Vlasov-Maxwell system (1.1), (1.2) was studied following different methods by DiPerna and Lions [7, Glassey and Schaeffer [9], 10], Glassey and

Received July 21, 2006.

2000 Mathematics Subject Classification. Primary 35A05, 35B35; Secondary 82D10.

Key words and phrases. Kinetic equations, Vlasov-Maxwell system, weak/mild solution, characteristics. E-mail address: mbostan@math.univ-fcomte.fr 
Strauss [11, [12, Klainerman and Staffilani [14, and Bouchut, Golse and Pallard 2]. Results for the boundary value problem were obtained by Poupaud [15], and Guo 13.

Here we intend to analyze a reduced 1D Vlasov-Maxwell system introduced recently in the physical literature for studying laser-plasma interactions. The assumptions of this model are the following : all unknowns depend on only one space variable, for example $x_{1}$, and the electrons are monokinetic in the directions transversal to $x_{1}$. The distribution function becomes

$$
F(t, x, p)=f\left(t, x_{1}, p_{1}\right) \delta\left(p_{2}-p_{2}\left(t, x_{1}\right)\right) \delta\left(p_{3}-p_{3}\left(t, x_{1}\right)\right) .
$$

We consider the initial condition

$$
F(0, x, p)=F_{0}(x, p):=f_{0}\left(x_{1}, p_{1}\right) \delta\left(p_{2}-p_{2}^{0}\left(x_{1}\right)\right) \delta\left(p_{3}-p_{3}^{0}\left(x_{1}\right)\right) .
$$

Let us also introduce the vector and scalar potentials $A, \Phi$ such that

$$
B=\operatorname{curl} A, \quad E=-\partial_{t} A-\nabla_{x} \Phi .
$$

Under the hypotheses of our model $\partial_{x_{2}}=\partial_{x_{3}}=0$ and thus the previous equalities become

$$
B_{1}=0, \quad B_{2}=-\partial_{x_{1}} A_{3}, \quad B_{3}=\partial_{x_{1}} A_{2}, E_{1}=-\partial_{t} A_{1}-\partial_{x_{1}} \Phi, \quad E_{2}=-\partial_{t} A_{2}, \quad E_{3}=-\partial_{t} A_{3} .
$$

The distribution function $f$ also satisfies a Vlasov equation in the phase space $\left(x_{1}, p_{1}\right)$. This comes mainly from the invariance of the quantities $P_{2}-e A_{2}, P_{3}-e A_{3}$ along the characteristics associated to (1.1)

$$
\begin{gathered}
\frac{d X}{d s}=v(P(s)), \frac{d P}{d s}=-e(E(s, X(s))+v(P(s)) \wedge B(s, X(s))), \\
X(s=t)=x, \quad P(s=t)=p .
\end{gathered}
$$

Assuming that $e A_{2}\left(0, x_{1}\right)=p_{2}^{0}\left(x_{1}\right), e A_{3}\left(0, x_{1}\right)=p_{3}^{0}\left(x_{1}\right)$ and $A_{1}=0$ we deduce after some computations (see [5]) that $f$ satisfies the kinetic equation

$$
\partial_{t} f+\frac{p_{1}}{m \gamma} \partial_{x_{1}} f-e\left(E_{1}\left(t, x_{1}\right)+\frac{e}{m \gamma} A_{2}\left(t, x_{1}\right) \partial_{x_{1}} A_{2}+\frac{e}{m \gamma} A_{3}\left(t, x_{1}\right) \partial_{x_{1}} A_{3}\right) \partial_{p_{1}} f=0,
$$

with the initial condition $f\left(0, x_{1}, p_{1}\right)=f_{0}\left(x_{1}, p_{1}\right), \quad\left(x_{1}, p_{1}\right) \in \mathbb{R}^{2}$ and we have the equality $F(t, x, p)=f\left(t, x_{1}, p_{1}\right) \delta\left(p_{2}-e A_{2}\left(t, x_{1}\right)\right) \delta\left(p_{3}-e A_{3}\left(t, x_{1}\right)\right)$. The function $\gamma$ is given by

$$
\gamma\left(t, x_{1}, p_{1}\right)=\left(1+\frac{\left|p_{1}\right|^{2}}{m^{2} c^{2}}+\frac{e^{2}}{m^{2} c^{2}}\left(\left|A_{2}\left(t, x_{1}\right)\right|^{2}+\left|A_{3}\left(t, x_{1}\right)\right|^{2}\right)\right)^{\frac{1}{2}} .
$$

For the sake of simplicity we assume that $A_{3}=0$. Under these circumstances, by adding the first and second Maxwell equations one gets the system

$$
\begin{gathered}
\partial_{t} f+\frac{p_{1}}{m \gamma} \partial_{x_{1}} f-e\left(E_{1}\left(t, x_{1}\right)+\frac{e}{m \gamma} A_{2}\left(t, x_{1}\right) \partial_{x_{1}} A_{2}\right) \partial_{p_{1}} f=0, \\
\partial_{t}^{2} A_{2}-c^{2} \partial_{x_{1}}^{2} A_{2}=-\frac{e^{2}}{m \varepsilon_{0}} \rho_{\gamma}\left(t, x_{1}\right) A_{2}\left(t, x_{1}\right), \\
\partial_{t} E_{1}=\frac{e}{\varepsilon_{0}} j_{1}\left(t, x_{1}\right), \\
\partial_{x_{1}} E_{1}=\frac{e}{\varepsilon_{0}}\left(\rho_{\text {ext }}\left(x_{1}\right)-\rho\left(t, x_{1}\right)\right),
\end{gathered}
$$


where $\left\{\rho, \rho_{\gamma}, j_{1}\right\}\left(t, x_{1}\right)=\int_{\mathbb{R}}\left\{1, \frac{1}{\gamma}, \frac{p_{1}}{\gamma}\right\} f\left(t, x_{1}, p_{1}\right) d p_{1}$, and $\gamma=\left(1+\frac{\left|p_{1}\right|^{2}}{m^{2} c^{2}}+\frac{e^{2}\left|A_{2}\left(t, x_{1}\right)\right|^{2}}{m^{2} c^{2}}\right)^{\frac{1}{2}}$. Observe also that the total energy at the moment $t$ is

$$
\begin{aligned}
& \int_{\mathbb{R}^{3}} \int_{\mathbb{R}^{3}} F(t, x, p) m c^{2}\left(1+\frac{|p|^{2}}{m^{2} c^{2}}\right)^{\frac{1}{2}} d p d x+\frac{\varepsilon_{0}}{2} \int_{\mathbb{R}^{3}}\left\{|E(t, x)|^{2}+c^{2}|B(t, x)|^{2}\right\} d x \\
& =\int_{\mathbb{R}} \int_{\mathbb{R}} f\left(t, x_{1}, p_{1}\right) m c^{2}\left(1+\frac{\left|p_{1}\right|^{2}}{m^{2} c^{2}}+\frac{e^{2}\left|A_{2}\left(t, x_{1}\right)\right|^{2}}{m^{2} c^{2}}\right)^{\frac{1}{2}} d p_{1} d x_{1} \\
& \quad+\frac{\varepsilon_{0}}{2} \int_{\mathbb{R}}\left\{\left|E_{1}\left(t, x_{1}\right)\right|^{2}+\left|\partial_{t} A_{2}\right|^{2}+c^{2}\left|\partial_{x_{1}} A_{2}\right|^{2}\right\} d x_{1} .
\end{aligned}
$$

The above model describes the interaction of the electro-magnetic field created by a laser wave (called pump wave) with a population of charged particles. It was studied recently by Carrillo and Labrunie in [5]. The strong nonlinear coupling through the Lorentz factor $\gamma$ makes this system difficult to study theoretically but also numerically. Other reduced models have been considered by physicists.

1) The nonrelativistic model NR is obtained by setting $\gamma=1$ everywhere.

2) The quasi-relativistic model (also called semi-relativistic by some authors) denoted QR consists in approximating $\gamma$ by $\left(1+\frac{\left|p_{1}\right|^{2}}{m^{2} c^{2}}\right)^{\frac{1}{2}}$ in the second term of (1.5) and in the definition of $j_{1}$, and setting $\gamma=1$ in the third term of (1.5) and in the definition of $\rho_{\gamma}$ (which means $\rho_{\gamma}=\rho$ ).

3) The original model with $\gamma=\left(1+\frac{\left|p_{1}\right|^{2}}{m^{2} c^{2}}+\frac{e^{2}\left|A_{2}\left(t, x_{1}\right)\right|^{2}}{m^{2} c^{2}}\right)^{\frac{1}{2}}$ will be referred to as fully relativistic FR.

Notice that $\left(F=f\left(t, x_{1}, p_{1}\right) \delta\left(p_{2}-e A_{2}\left(t, x_{1}\right)\right) \delta\left(p_{3}\right), E, B\right)$, where $\left(f, E_{1}, A_{2}\right)$ solves the NR model (1.5), (1.6), (1.7), (1.8), is a class of exact solutions for the nonrelativistic Vlasov-Maxwell system, i.e., (1.1), (1.2) with $v(p)=\frac{p}{m}$. Similarly, when $\left(f, E_{1}, A_{2}\right)$ solves the FR model, then $\left(F=f\left(t, x_{1}, p_{1}\right) \delta\left(p_{2}-e A_{2}\left(t, x_{1}\right)\right) \delta\left(p_{3}\right), E, B\right)$ is a class of exact solutions for the relativistic Vlasov-Maxwell system (1.1), (1.2). Nevertheless, the QR model is only an approximation of the FR model.

Another way to retrieve the FR model is to consider particular solutions of the one and one-half relativistic Vlasov-Maxwell system

$$
\begin{gathered}
\partial_{t} \tilde{F}+\frac{p_{1}}{m \tilde{\gamma}} \partial_{x_{1}} \tilde{F}-e\left(E_{1}+\frac{p_{2}}{m \tilde{\gamma}} B_{3}\right) \partial_{p_{1}} \tilde{F}-e\left(E_{2}-\frac{p_{1}}{m \tilde{\gamma}} B_{3}\right) \partial_{p_{2}} \tilde{F}=0, \\
\partial_{t} E_{1}=\frac{e}{\varepsilon_{0}} \int_{\mathbb{R}^{2}} \frac{p_{1}}{m \tilde{\gamma}} \tilde{F} d p, \partial_{t} E_{2}+c^{2} \partial_{x_{1}} B_{3}=\frac{e}{\varepsilon_{0}} \int_{\mathbb{R}^{2}} \frac{p_{2}}{m \tilde{\gamma}} \tilde{F} d p, \partial_{t} B_{3}+\partial_{x_{1}} E_{2}=0,
\end{gathered}
$$

where $\tilde{\gamma}=\left(1+\frac{\left|p_{1}\right|^{2}+\left|p_{2}\right|^{2}}{m^{2} c^{2}}\right)^{\frac{1}{2}}$. Let us introduce the vector potential component $A_{2}$ such that $E_{2}=-\partial_{t} A_{2}, B_{3}=\partial_{x_{1}} A_{2}$. By direct computation we find as before that the solution of the one and one-half relativistic Vlasov-Maxwell system corresponding to the initial particle distribution $\tilde{F}\left(0, x_{1}, p_{1}, p_{2}\right)=f_{0}\left(x_{1}, p_{1}\right) \delta\left(p_{2}-e A_{2}\left(0, x_{1}\right)\right)$ is related to the solution of the FR model by

$$
\tilde{F}\left(t, x_{1}, p_{1}, p_{2}\right)=f\left(t, x_{1}, p_{1}\right) \delta\left(p_{2}-e A_{2}\left(t, x_{1}\right)\right) .
$$


Let us mention that the existence of classical solutions for (1.9), (1.10) was established in 9]. The authors construct global $C^{1}$ solutions for compactly supported, smooth initial conditions. The aim of this paper is to provide existence and uniqueness results for the FR model. Motivated by the delta function appearing in (1.11) it is natural to consider weak solutions of the Vlasov equation. More precisely we are looking for solutions by characteristics. We also remove the hypotheses on the compactness of the supports, which do not correspond to the physical reality. It is worth pointing out that this hypothesis together with the smoothness of the particle distribution were crucial for the arguments in 9. Although the FR model can be interpreted as a particular case of the one and one-half relativistic Vlasov-Maxwell system, here we employ a different method than thoses in [9]. Our proof makes no appeal to the decomposition of derivatives, which is one of the main tools in [9]. At this stage let us mention the recent analysis of the relativistic Vlasov-Maxwell equations in [8] where the authors study particle distributions of bounded variation.

The equations (1.5), (1.6), (1.7), (1.8) can be simplified by introducing dimensionless unknowns and variables. If we omit the subscripts of $x_{1}, p_{1}, E_{1}, A_{2}, j_{1}$ and keep the same notations for the rescaled unknowns and variables we obtain (think that $m=1, c=$ $\left.1, e=1, \varepsilon_{0}=1\right)$

$$
\begin{gathered}
\partial_{t} f+\frac{p}{\gamma_{1}} \partial_{x} f-\left(E(t, x)+\frac{A(t, x)}{\gamma_{2}} \partial_{x} A\right) \partial_{p} f=0, \\
\partial_{t}^{2} A-\partial_{x}^{2} A=-\rho_{\gamma_{2}}(t, x) A(t, x), \\
\partial_{t} E=j(t, x), \\
\partial_{x} E=\rho_{\text {ext }}(x)-\rho(t, x),
\end{gathered}
$$

where $\left\{\rho, \rho_{\gamma_{2}}, j\right\}(t, x)=\int_{\mathbb{R}}\left\{1, \frac{1}{\gamma_{2}}, \frac{p}{\gamma_{1}}\right\} f(t, x, p) d p, \gamma_{1}=\gamma_{2}=1$ in the NR case, $\gamma_{1}=$ $\left(1+|p|^{2}\right)^{1 / 2}, \gamma_{2}=1$ in the QR case and $\gamma_{1}=\gamma_{2}=\left(1+|p|^{2}+|A(t, x)|^{2}\right)^{1 / 2}$ in the FR case. We supplement these equations with initial conditions

$$
f(0, x, p)=f_{0}(x, p),(x, p) \in \mathbb{R}^{2}, \quad\left(E, A, \partial_{t} A\right)(0, x)=\left(E_{0}, A_{0}, A_{1}\right)(x), x \in \mathbb{R} .
$$

In [5] the authors investigated the existence of space periodic solutions and free-space solutions of the system (1.5), (1.6), (1.7), (1.8). They proved the existence of weak and characteristic solutions in the NR and QR cases. In this article we restrict our attention to the FR case. Actually the same method applies to the QR case, and some arguments can be used for analyzing the NR case. It is possible to construct global solutions by characteristics in the QR and FR cases, while only local solutions by characteristics are available in the NR case (see also [5]). The arguments rely on iterative procedure (cf. [6]). The main idea consists in using the formulation by characteristics to obtain $L^{\infty}$ estimates for the electro-magnetic field and the spacial derivatives by duality computations involving $L^{1}$ test functions. This method has already been used in 1 to prove the existence and uniqueness of the solution by characteristics for the 1D Vlasov-Poisson initial-boundary value problem.

To our knowledge this is the first theoretical work on the FR reduced Vlasov-Maxwell model (1.5), (1.6), (1.7), (1.8). It has common features with the Nordström-Vlasov system, studied recently by Calogero and Rein [3, 4]. 
The paper is organized as follows. In Section 2 we recall the notion of weak and mild solutions for the Vlasov problem and several properties of such solutions. We establish the continuous dependence of the characteristics upon the electro-magnetic field and the initial conditions. In Section 3 we define the fixed point application $(E, A) \rightarrow$ $\mathcal{F}(E, A)$ for regular fields $(E, A)$ and we construct a domain $D$ which is left invariant by this application. The main ingredient for using the iteration method is to estimate $\mathcal{F}\left(E_{1}, A_{1}\right)-\mathcal{F}\left(E_{2}, A_{2}\right)$ in terms of $\left(E_{1}, A_{1}\right)-\left(E_{2}, A_{2}\right)$ for pairs $\left(E_{1}, A_{1}\right),\left(E_{2}, A_{2}\right) \in D$. In the next section we prove the existence of a unique fixed point for $\mathcal{F}$ which guarantees the existence and uniqueness of a solution for the reduced Vlasov-Maxwell system. This solution preserves the total energy.

2. The Vlasov problem. In this section we assume that the fields $E, A$ are given and we introduce the notions of weak solution and mild solution (or solution by characteristics). We easily check that in all three cases we have $\operatorname{div}_{(x, p)}\left(\frac{p}{\gamma_{1}},-E(t, x)-\frac{A(t, x)}{\gamma_{2}} \partial_{x} A\right)=$ 0 , and therefore the Vlasov equation (1.12) can also be written

$$
\left.\partial_{t} f+\partial_{x}\left(\frac{p}{\gamma_{1}} f\right)-\partial_{p}\left(\left(E(t, x)+\frac{A(t, x)}{\gamma_{2}} \partial_{x} A\right) f\right)=0, \quad(t, x, p) \in\right] 0, T\left[\times \mathbb{R}^{2} .\right.
$$

Consider the initial condition

$$
f(0, x, p)=f_{0}(x, p), \quad(x, p) \in \mathbb{R}^{2} .
$$

Definition 2.1. Assume that $E \in L^{\infty}(] 0, T[\times \mathbb{R}), A \in L^{\infty}(] 0, T\left[; W^{1, \infty}(\mathbb{R})\right), f_{0} \in$ $L_{\text {loc }}^{1}\left(\mathbb{R}^{2}\right)$. We say that $f \in L_{\text {loc }}^{1}\left(\left[0, T\left[\times \mathbb{R}^{2}\right)\right.\right.$ is a weak solution for the Vlasov problem (2.1), (2.2) iff

$$
\begin{array}{r}
-\int_{0}^{T} \int_{\mathbb{R}} \int_{\mathbb{R}} f(t, x, p)\left(\partial_{t} \varphi+\frac{p}{\gamma_{1}} \partial_{x} \varphi-(E(t, x)\right. \\
\left.\left.+\frac{A(t, x)}{\gamma_{2}} \partial_{x} A\right) \partial_{p} \varphi\right) d p d x d t \\
=\int_{\mathbb{R}} \int_{\mathbb{R}} f_{0}(x, p) \varphi(0, x, p) d p d x
\end{array}
$$

for all test functions $\varphi \in C_{c}^{1}\left(\left[0, T\left[\times \mathbb{R}^{2}\right)\right.\right.$.

We consider some special solutions of (2.1), (2.2) which are called mild solutions or solutions by characteristics. These solutions require more regularity for $E, A$. Assume that $E \in L^{\infty}(] 0, T\left[; W^{1, \infty}(\mathbb{R})\right), A \in L^{\infty}(] 0, T\left[; W^{2, \infty}(\mathbb{R})\right)$ and let us introduce the system of characteristics associated to (1.12)

$$
\frac{d X}{d s}=\frac{P(s)}{\gamma_{1}}, \frac{d P}{d s}=-E(x, X(s))-\frac{A(s, X(s))}{\gamma_{2}} \partial_{x} A(s, X(s)),
$$

with the initial conditions

$$
X(s=t)=x, P(s=t)=p .
$$

Observe that under the above regularity hypotheses for $E, A$, for all $(t, x, p) \in\left[0, T\left[\times \mathbb{R}^{2}\right.\right.$ there is a unique solution for (2.4), (2.5) denoted $(X(s), P(s))=(X(s ; t, x, p), P(s ; t, x, p))$. 
The definition of the solution by characteristics can be obtained by replacing the transport term of equation (2.3) by a test function $\psi$ :

$$
\partial_{t} \varphi+\frac{p}{\gamma_{1}} \partial_{x} \varphi-\left(E(t, x)+\frac{A(t, x)}{\gamma_{2}} \partial_{x} A\right) \partial_{p} \varphi=-\psi .
$$

After integration along the characteristics and by imposing $\varphi(T, \cdot, \cdot)=0$ we find formally that $\varphi(t, x, p)=\int_{t}^{T} \psi(s, X(s ; t, x, p), P(s ; t, x, p)) d s$.

Definition 2.2. Assume that $E \in L^{\infty}(] 0, T\left[; W^{1, \infty}(\mathbb{R})\right), A \in L^{\infty}(] 0, T\left[; W^{2, \infty}(\mathbb{R})\right)$, $f_{0} \in L_{\text {loc }}^{1}\left(\mathbb{R}^{2}\right)$. We say that $f \in L_{\text {loc }}^{1}\left([0, T] \times \mathbb{R}^{2}\right)$ is a mild solution for the Vlasov problem (2.1), (2.2) iff

$$
\int_{0}^{T} \int_{\mathbb{R}} \int_{\mathbb{R}} f \psi d p d x d t=\int_{\mathbb{R}} \int_{\mathbb{R}} f_{0}(x, p) \int_{0}^{T} \psi(s, X(s ; 0, x, p), P(s ; 0, x, p)) d s d p d x,
$$

for all test functions $\psi \in C_{c}^{0}\left([0, T] \times \mathbb{R}^{2}\right)$.

It is well known that the mild solution is unique and is given by

$$
f(t, x, p)=f_{0}(X(0 ; t, x, p), P(0 ; t, x, p)), \quad \forall(t, x, p) \in[0, T] \times \mathbb{R}^{2} .
$$

It is easily seen that any mild solution is also a weak solution. By performing the change of variables $(x, p) \rightarrow(X(t ; 0, x, p), P(t ; 0, x, p))$ we verify that if $f_{0} \in L^{1}\left(\mathbb{R}^{2}\right)$, then the mild solution belongs to $L^{\infty}(] 0, T\left[; L^{1}\left(\mathbb{R}^{2}\right)\right)$ and

$$
\left.\int_{\mathbb{R}} \int_{\mathbb{R}}|f(t, x, p)| d p d x=\int_{\mathbb{R}} \int_{\mathbb{R}}\left|f_{0}(x, p)\right| d p d x, \quad \forall t \in\right] 0, T[.
$$

Obviously, if $f_{0}$ is nonnegative, $f$ remains nonnegative. Note also that if $f_{0}$ belongs to $L^{1}\left(\mathbb{R}^{2}\right)$, then the mild formulation holds true for any continuous bounded test function $\psi \in C^{0}\left([0, T] \times \mathbb{R}^{2}\right) \cap L^{\infty}(] 0, T\left[\times \mathbb{R}^{2}\right)$.

2.1. Continuous dependence of characteristics. Here we estimate the difference between two solutions of the characteristic system (2.4), (2.5). The proof is immediate and is left to the reader.

Proposition 2.3. Assume that $E, \tilde{E}$ belong to $L^{\infty}(] 0, T\left[; W^{1, \infty}(\mathbb{R})\right), A, \tilde{A}$ belong to $L^{\infty}(] 0, T\left[; W^{2, \infty}(\mathbb{R})\right)$ and consider $(t, x, p),(t, \tilde{x}, \tilde{p}) \in[0, T] \times \mathbb{R}^{2}$. We denote by $(X, P)(s ; t, x, p)$, resp. $(\tilde{X}, \tilde{P})(s ; t, \tilde{x}, \tilde{p})$, the solution of (2.4), (2.5) corresponding to $(E, A),(\tilde{E}, \tilde{A})$.

1) In the NR and QR cases we have for all $s \in[0, T]$

$$
\begin{aligned}
& \left(|X(s)-\tilde{X}(s)|^{2}+|P(s)-\tilde{P}(s)|^{2}\right)^{\frac{1}{2}} \leq\left\{\left(|x-\tilde{x}|^{2}+|p-\tilde{p}|^{2}\right)^{\frac{1}{2}}\right. \\
+ & \left.\left|\int_{t}^{s}\left(\|(E-\tilde{E})(\tau)\|_{\infty}+\left\|(A-\tilde{A}) \partial_{x} A(\tau)\right\|_{\infty}+\left\|\tilde{A}\left(\partial_{x} A-\partial_{x} \tilde{A}\right)(\tau)\right\|_{\infty}\right) d \tau\right|\right\} \\
\times & \exp \left(\left|\int_{t}^{s}\left\{1+\left\|\partial_{x} E(\tau)\right\|_{\infty}+\left\|\partial_{x} A(\tau)\right\|_{\infty}^{2}+\left\|A \partial_{x}^{2} A(\tau)\right\|_{\infty}\right\} d \tau\right|\right) .
\end{aligned}
$$


2) In the FR case we have for all $s \in[0, T]$

$$
\begin{aligned}
& \left(|X(s)-\tilde{X}(s)|^{2}+|P(s)-\tilde{P}(s)|^{2}\right)^{\frac{1}{2}} \leq\left\{\left(|x-\tilde{x}|^{2}+|p-\tilde{p}|^{2}\right)^{\frac{1}{2}}\right. \\
+ & \left.C\left|\int_{t}^{s}\left(\|(E-\tilde{E})(\tau)\|_{\infty}+\left(1+\left\|\partial_{x} A\right\|_{\infty}\right)\|(A-\tilde{A})(\tau)\|_{\infty}+\left\|\left(\partial_{x} A-\partial_{x} \tilde{A}\right)(\tau)\right\|_{\infty}\right) d \tau\right|\right\} \\
\times & \exp \left(C\left|\int_{t}^{s}\left\{1+\left\|\partial_{x} E(\tau)\right\|_{\infty}+\left\|\partial_{x} A(\tau)\right\|_{\infty}^{2}+\left\|\partial_{x}^{2} A(\tau)\right\|_{\infty}\right\} d \tau\right|\right) .
\end{aligned}
$$

3. Existence and uniqueness for the reduced model. We intend to prove the existence and uniqueness of the mild solution for the system (1.12), (1.13), (1.14), (1.15), (1.16) in the FR case by using the iterated approximation method. We assume that $\gamma_{1}=\gamma_{2}=\left(1+|p|^{2}+|A(t, x)|^{2}\right)^{1 / 2}$ everywhere from now on if nothing else specified. Nevertheless we prefer to distinguish the Lorentz factors $\gamma_{1}, \gamma_{2}$; the reader can try to adapt the proofs in order to treat the NR and QR cases. We consider the application $\mathcal{F}$ defined for regular fields $E \in L^{\infty}(] 0, T\left[; W^{1, \infty}(\mathbb{R})\right)$ and $A \in L^{\infty}(] 0, T\left[; W^{2, \infty}(\mathbb{R})\right)$ by $(E, A) \rightarrow f_{E, A} \rightarrow(\tilde{E}, \tilde{A})=: \mathcal{F}(E, A)$. Here $f_{E, A}$ is the mild solution of the Vlasov problem (2.1), (2.2) associated with the fields $E, A$, and $\tilde{E}, \tilde{A}$ are given by

$$
\int_{\mathbb{R}} \tilde{E}(t, x) \varphi(x) d x=\int_{\mathbb{R}} E_{0}(x) \varphi(x) d x+\int_{\mathbb{R}} \int_{\mathbb{R}} f_{0}(x, p) \int_{x}^{X(t ; 0, x, p)} \varphi(u) d u d p d x
$$

for any function $\varphi \in L^{1}(\mathbb{R})$, where $(X, P)$ are the characteristics associated with $(E, A)$, respectively

$$
\begin{aligned}
\tilde{A}(t, x) & =\frac{1}{2}\left(A_{0}(x+t)+A_{0}(x-t)\right)+\frac{1}{2} \int_{x-t}^{x+t} A_{1}(y) d y \\
& -\frac{1}{2} \int_{0}^{t} \int_{x-(t-s)}^{x+(t-s)}\left(\rho_{\gamma_{2}} A\right)(s, y) d y d s, \quad(t, x) \in[0, T] \times \mathbb{R},
\end{aligned}
$$

where $\rho_{\gamma_{2}}(t, x)=\int_{\mathbb{R}} \frac{f_{E, A}(t, x, p)}{\gamma_{2}} d p$. Obviously the expression of $\tilde{A}$ comes from the Duhamel representation formula for the solution of the wave equation in one dimension with the source term $-\rho_{\gamma_{2}} A$ and the initial conditions $A_{0}, A_{1}$. Note that if $A_{1} \in L^{\infty}(\mathbb{R})$, $f_{0} \in L^{1}\left(\mathbb{R}^{2}\right)$, then $\rho_{\gamma_{2}} \in L^{\infty}(] 0, T\left[; L^{1}(\mathbb{R})\right)$ which implies that $\rho_{\gamma_{2}} A \in L^{\infty}(] 0, T\left[; L^{1}(\mathbb{R})\right)$ and thus $\tilde{A}$ is well defined. Let us now explain our choice for the definition of $\tilde{E}$. The Maxwell equations involving the electrostatic field $\tilde{E}$ are

$$
\left.\partial_{t} \tilde{E}=j_{E, A}=\int_{\mathbb{R}} \frac{p}{\gamma_{1}} f_{E, A} d p, \quad \partial_{x} \tilde{E}=\rho_{\text {ext }}-\rho_{E, A}=\rho_{\text {ext }}-\int_{\mathbb{R}} f_{E, A} d p, \quad(t, x) \in\right] 0, T[\times \mathbb{R},
$$

with the initial condition $\tilde{E}(0, x)=E_{0}(x), \quad x \in \mathbb{R}$. By using the continuity equation $\partial_{t} \rho_{E, A}+\partial_{x} j_{E, A}=0$ it is sufficient to impose $E_{0}^{\prime}=\rho_{e x t}-\rho_{0}$ where $\rho_{0}=\int_{\mathbb{R}} f_{0} d p$ and to solve $\partial_{t} \tilde{E}=j_{E, A}$, which gives $\tilde{E}(t, x)=E_{0}(x)+\int_{0}^{t} j_{E, A}(s, x) d s$. After multiplication by a test function $\varphi \in L^{1}(\mathbb{R})$ one gets by formal computations using the mild formulation 
(2.6)

$$
\begin{aligned}
\int_{\mathbb{R}} \tilde{E}(t, x) \varphi(x) d x & =\int_{\mathbb{R}} E_{0}(x) \varphi(x) d x+\int_{0}^{t} \int_{\mathbb{R}} \int_{\mathbb{R}} f_{E, A}(s, x, p) \frac{p}{\gamma_{1}} \varphi(x) d p d x d s \\
& =\int_{\mathbb{R}} E_{0}(x) \varphi(x) d x+\int_{\mathbb{R}} \int_{\mathbb{R}} f_{0}(x, p) \int_{0}^{t} \frac{d X}{d s} \varphi(X(s)) d s d p d x \\
& =\int_{\mathbb{R}} E_{0}(x) \varphi(x) d x+\int_{\mathbb{R}} \int_{\mathbb{R}} f_{0}(x, p) \int_{x}^{X(t ; 0, x, p)} \varphi(u) d u d p d x
\end{aligned}
$$

Note that if $E_{0} \in L^{\infty}(\mathbb{R})$ and $f_{0} \in L^{1}\left(\mathbb{R}^{2}\right)$ the formula (3.1) defines a unique $\tilde{E} \in$ $L^{\infty}(] 0, T[\times \mathbb{R})$ and $\left.\|\tilde{E}(t)\|_{L^{\infty}(\mathbb{R})} \leq\left\|E_{0}\right\|_{L^{\infty}(\mathbb{R})}+\left\|f_{0}\right\|_{L^{1}\left(\mathbb{R}^{2}\right)}, \quad t \in\right] 0, T[$. The idea is to study the existence and uniqueness of a fixed point for $\mathcal{F}$. We introduce some notations. If $u: \mathbb{R} \rightarrow\left[0,+\infty\left[\right.\right.$ is a bounded function nondecreasing on $\mathbb{R}^{-}$and nonincreasing on $\mathbb{R}^{+}$and $R>0$, we denote by $u^{R}: \mathbb{R} \rightarrow\left[0,+\infty\left[\right.\right.$ the function given by $u^{R}(t)=u(0)$ if $|t| \leq R, u^{R}(t)=u(t-R)$ if $t>R$ and $u^{R}(t)=u(t+R)$ if $t<-R$. If $u \in L^{1}(\mathbb{R})$, then $u^{R} \in L^{1}(\mathbb{R})$ and $\left\|u^{R}\right\|_{L^{1}(\mathbb{R})}=2 R\|u\|_{L^{\infty}(\mathbb{R})}+\|u\|_{L^{1}(\mathbb{R})}$. Following the ideas in [5] we obtain $L^{\infty}$ bounds for $\tilde{E}, \tilde{A}$ and their first derivatives.

3.1. Estimates for $\tilde{E}$. We assume that $f_{0}$ verifies the following hypotheses : there is $n_{0}: \mathbb{R} \rightarrow\left[0,+\infty\left[\right.\right.$ nondecreasing on $\mathbb{R}^{-}$and nonincreasing on $\mathbb{R}^{+}$such that

$$
\begin{gathered}
(H) f_{0}(x, p) \leq n_{0}(p), \quad \forall(x, p) \in \mathbb{R}^{2}, \\
\left(H_{0}\right) \quad M_{0}:=\int_{\mathbb{R}} n_{0}(p) d p<+\infty, \\
\left(H_{\infty}\right) \quad M_{\infty}:=\left\|n_{0}\right\|_{L^{\infty}(\mathbb{R})}<+\infty .
\end{gathered}
$$

We can prove the following $L^{\infty}$ bounds for $\tilde{E}, \partial_{x} \tilde{E}$.

Proposition 3.1. Assume that $f_{0}$ is nonnegative, belongs to $L^{1}\left(\mathbb{R}^{2}\right)$ and satisfies $(H)$, $\left(H_{0}\right),\left(H_{\infty}\right)$. We also suppose that $\rho_{\text {ext }}$ is a given nonnegative function in $L^{1}(\mathbb{R}) \cap L^{\infty}(\mathbb{R})$ and that $E_{0}$ is a primitive of $\rho_{\text {ext }}-\rho_{0}$, where $\rho_{0}=\int_{\mathbb{R}} f_{0} d p$. Then for all regular fields $E \in L^{\infty}(] 0, T\left[; W^{1, \infty}(\mathbb{R})\right), A \in L^{\infty}(] 0, T\left[; W^{2, \infty}(\mathbb{R})\right)$ we have $\tilde{E} \in L^{\infty}(] 0, T\left[; W^{1, \infty}(\mathbb{R})\right)$, and the following estimates hold for all $t \in[0, T]$ :

$$
\begin{gathered}
\|\tilde{E}\|_{L^{\infty}(\mathbb{R})} \leq\left\|E_{0}\right\|_{L^{\infty}(\mathbb{R})}+\left\|f_{0}\right\|_{L^{1}\left(\mathbb{R}^{2}\right)} \\
\left\|\partial_{x} \tilde{E}\right\|_{L^{\infty}(\mathbb{R})} \leq\left\|\rho_{e x t}\right\|_{L^{\infty}(\mathbb{R})}+M_{0}+2 M_{\infty} \int_{0}^{t}\left\{\|E(s)\|_{L^{\infty}(\mathbb{R})}+\left\|A(s) \partial_{x} A(s)\right\|_{L^{\infty}(\mathbb{R})}\right\} d s
\end{gathered}
$$

Proof. The estimate (3.6) follows immediately from our definition for $\tilde{E}$. We introduce the charge density $\rho_{E, A}=\int_{\mathbb{R}} f_{E, A} d p$. By using (2.7) we can write

$$
\rho_{E, A}(t, x)=\int_{\mathbb{R}} f_{0}(X(0 ; t, x, p), P(0 ; t, x, p)) d p \leq \int_{\mathbb{R}} n_{0}(P(0 ; t, x, p)) d p .
$$

Now using the second equation in (2.4) yields

$$
|P(0 ; t, x, p)-p| \leq \int_{0}^{t}\left\{\|E(s)\|_{L^{\infty}(\mathbb{R})}+\left\|A(s) \partial_{x} A(s)\right\|_{L^{\infty}(\mathbb{R})}\right\} d s=: R(t) .
$$


Notice that if $p>R(t)$, then $P(0 ; t, x, p) \geq p-R(t)>0$ and thus $n_{0}(P(0 ; t, x, p)) \leq$ $n_{0}(p-R(t))=n_{0}^{R(t)}(p)$. Similarly, if $p<-R(t)$, then $P(0 ; t, x, p) \leq p+R(t)<0$ and thus $n_{0}(P(0 ; t, x, p)) \leq n_{0}(p+R(t))=n_{0}^{R(t)}(p)$. When $|p| \leq R(t)$ we obviously have $n_{0}(P(0 ; t, x, p)) \leq n_{0}(0)=n_{0}^{R(t)}(p)$. Therefore we obtain

$$
\rho_{E, A}(t, x) \leq \int_{\mathbb{R}} n_{0}^{R(t)}(p) d p=2 R(t) M_{\infty}+M_{0}, \quad(t, x) \in[0, T] \times \mathbb{R} .
$$

In order to estimate $\partial_{x} \tilde{E}$ we prove that $\tilde{E}$ satisfies $\partial_{x} \tilde{E}=\rho_{e x t}-\rho_{E, A}$. Indeed, take $\varphi \in C_{c}^{1}(\mathbb{R})$ and let us calculate

$$
\begin{aligned}
\int_{\mathbb{R}} \tilde{E}(t, x) \frac{d \varphi}{d x} d x & =-\int_{\mathbb{R}} E_{0}^{\prime} \varphi(x) d x+\int_{\mathbb{R}} \int_{\mathbb{R}} f_{0}(x, p)(\varphi(X(t ; 0, x, p))-\varphi(x)) d p d x \\
& =-\int_{\mathbb{R}}\left(\rho_{\text {ext }}(x)-\rho_{E, A}(t, x)\right) \varphi(x) d x .
\end{aligned}
$$

Therefore we have $\partial_{x} \tilde{E}(t)=\rho_{\text {ext }}-\rho_{E, A}(t)$, and (3.7) follows by (3.8).

3.2. Estimates for $\tilde{A}$. We now establish that $L^{\infty}$ bounds for $\tilde{A}, \partial_{x} \tilde{A}$ and $\partial_{t} \tilde{A}$.

Proposition 3.2. Assume that $f_{0}$ is nonnegative, belongs to $L^{1}\left(\mathbb{R}^{2}\right)$ and satisfies $(H),\left(H_{0}\right),\left(H_{\infty}\right)$. We suppose also that $A_{0} \in W^{1, \infty}(\mathbb{R}), A_{1} \in L^{\infty}(\mathbb{R})$. Then for all regular fields $E \in L^{\infty}(] 0, T\left[; W^{1, \infty}(\mathbb{R})\right), A \in L^{\infty}(] 0, T\left[; W^{2, \infty}(\mathbb{R})\right)$ we have $\tilde{A} \in W^{1, \infty}(] 0, T[\times \mathbb{R})$ and

$$
\begin{gathered}
\|\tilde{A}(t)\|_{L^{\infty}(\mathbb{R})} \leq\left\|A_{0}\right\|_{L^{\infty}(\mathbb{R})}+t\left\|A_{1}\right\|_{L^{\infty}(\mathbb{R})}+\frac{\left\|f_{0}\right\|_{L^{1}\left(\mathbb{R}^{2}\right)}}{2} \int_{0}^{t}\|A(s)\|_{L^{\infty}(\mathbb{R})} d s, \quad t \in[0, T], \\
\max \left\{\left\|\partial_{x} \tilde{A}(t)\right\|_{L^{\infty}},\left\|\partial_{t} \tilde{A}\right\|_{L^{\infty}}\right\} \leq\left\|A_{0}^{\prime}\right\|_{L^{\infty}}+\left\|A_{1}\right\|_{L^{\infty}}+\int_{0}^{t}\|A(s)\|_{L^{\infty}}\left(M_{0}+2 R(s) M_{\infty}\right) d s,
\end{gathered}
$$

where $R(t)=\int_{0}^{t}\left\{\|E(s)\|_{L^{\infty}(\mathbb{R})}+\left\|A(s) \partial_{x} A(s)\right\|_{L^{\infty}(\mathbb{R})}\right\} d s, \quad t \in[0, T]$.

Proof. From (3.2) we deduce easily that

$$
\begin{aligned}
\|\tilde{A}(t)\|_{L^{\infty}} & \leq\left\|A_{0}\right\|_{L^{\infty}}+t\left\|A_{1}\right\|_{L^{\infty}}+\frac{1}{2} \int_{0}^{t}\|A(s)\|_{L^{\infty}}\left\|\rho_{E, A}(s)\right\|_{L^{1}} d s \\
& \leq\left\|A_{0}\right\|_{L^{\infty}}+t\left\|A_{1}\right\|_{L^{\infty}}+\frac{\left\|f_{0}\right\|_{L^{1}\left(\mathbb{R}^{2}\right)}}{2} \int_{0}^{t}\|A(s)\|_{L^{\infty}(\mathbb{R})} d s .
\end{aligned}
$$

We have the following representation formula for the space derivative of $\tilde{A}$ :

$$
\begin{aligned}
\partial_{x} \tilde{A}(t, x) & =\frac{1}{2}\left\{A_{0}^{\prime}(x+t)+A_{0}^{\prime}(x-t)\right\}+\frac{1}{2}\left\{A_{1}(x+t)-A_{1}(x-t)\right\} \\
& -\frac{1}{2} \int_{0}^{t}\left\{\left(\rho_{\gamma_{2}} A\right)(s, x+t-s)-\left(\rho_{\gamma_{2}} A\right)(s, x-t+s)\right\} d s,
\end{aligned}
$$

and therefore, by using (3.8) we obtain the estimate

$$
\begin{aligned}
\left\|\partial_{x} \tilde{A}(t)\right\|_{L^{\infty}} & \leq\left\|A_{0}^{\prime}\right\|_{L^{\infty}}+\left\|A_{1}\right\|_{L^{\infty}}+\int_{0}^{t}\left\|A(s) \rho_{E, A}(s)\right\|_{L^{\infty}} d s \\
& \leq\left\|A_{0}^{\prime}\right\|_{L^{\infty}}+\left\|A_{1}\right\|_{L^{\infty}}+\int_{0}^{t}\|A(s)\|_{L^{\infty}}\left\{M_{0}+2 R(s) M_{\infty}\right\} d s .
\end{aligned}
$$


The time derivative of $\tilde{A}$ is given by

$$
\begin{aligned}
\partial_{t} \tilde{A}(t, x) & =\frac{1}{2}\left\{A_{0}^{\prime}(x+t)-A_{0}^{\prime}(x-t)\right\}+\frac{1}{2}\left\{A_{1}(x+t)+A_{1}(x-t)\right\} \\
& -\frac{1}{2} \int_{0}^{t}\left\{\left(\rho_{\gamma_{2}} A\right)(s, x+t-s)+\left(\rho_{\gamma_{2}} A\right)(s, x-t+s)\right\} d s,
\end{aligned}
$$

and we obtain the same estimate for $\partial_{t} \tilde{A}$ as for $\partial_{x} \tilde{A}$.

We now construct a domain $D_{T}$ for the application $\mathcal{F}$ such that

$$
\|\tilde{E}\|_{L^{\infty}(] 0, T\left[; W^{1, \infty}(\mathbb{R})\right)}+\|\tilde{A}\|_{W^{1, \infty}(] 0, T[\times \mathbb{R})} \leq C, \quad \forall(E, A) \in D_{T}
$$

for some constant depending only on the initial conditions and $T$.

Proposition 3.3. Assume that the hypotheses of Propositions 3.1, 3.2 hold. We consider the set

$$
\begin{aligned}
D_{T}=\left\{(E, A) \in L^{\infty}(] 0, T\left[; W^{1, \infty}(\mathbb{R})\right) \times L^{\infty}(] 0, T\left[; W^{2, \infty}(\mathbb{R})\right) \mid\|E\|_{L^{\infty}} \leq e,\right. \\
\left.\left\|\partial_{x} E\right\|_{L^{\infty}} \leq e_{1},\|A(t)\|_{L^{\infty}} \leq a(t),\left\|\partial_{x} A(t)\right\|_{L^{\infty}} \leq a_{1}(t), t \in[0, T]\right\},
\end{aligned}
$$

where $e=\left\|E_{0}\right\|_{L^{\infty}}+\left\|f_{0}\right\|_{L^{1}}, a(t)=\left(\left\|A_{0}\right\|_{L^{\infty}}+t\left\|A_{1}\right\|_{L^{\infty}}\right) \exp \left(\frac{t\left\|f_{0}\right\|_{L^{1}}}{2}\right), a_{1}(t)=$ $\left\{\left\|A_{0}^{\prime}\right\|_{L^{\infty}}+\left\|A_{1}\right\|_{L^{\infty}}+M_{0} t a(t)+M_{\infty} e t^{2} a(t)\right\} \exp \left(2 M_{\infty} t^{2} a(t)^{2}\right), t \in[0, T]$ and $e_{1}=$ $\left\|\rho_{\text {ext }}\right\|_{L^{\infty}}+M_{0}+2 M_{\infty} e T+2 M_{\infty} \int_{0}^{T} a(t) a_{1}(t) d t$. Then for all $(E, A) \in D_{T}$ and any $t \in[0, T]$ we have the inequalities

$$
\begin{aligned}
& \|\tilde{E}\|_{L^{\infty}} \leq e, \quad\left\|\partial_{x} \tilde{E}\right\|_{L^{\infty}} \leq e_{1}, \\
& \|\tilde{A}(t)\|_{L^{\infty}} \leq a(t), \quad \max \left\{\left\|\partial_{x} \tilde{A}(t)\right\|_{L^{\infty}},\left\|\partial_{t} \tilde{A}(t)\right\|_{L^{\infty}}\right\} \leq a_{1}(t), \quad t \in[0, T] .
\end{aligned}
$$

Proof. From (3.6) we have $\|\tilde{E}\|_{L^{\infty}} \leq e$. From (3.10) we obtain

$$
\begin{aligned}
\|\tilde{A}(t)\|_{L^{\infty}} & \leq\left(\left\|A_{0}\right\|_{L^{\infty}}+t\left\|A_{1}\right\|_{L^{\infty}}\right)\left(1+\frac{\left\|f_{0}\right\|_{L^{1}}}{2} \int_{0}^{t} \exp \left(\frac{s\left\|f_{0}\right\|_{L^{1}}}{2}\right) d s\right) \\
& =a(t) .
\end{aligned}
$$

We introduce the notation $c(t)=\left\|A_{0}^{\prime}\right\|_{L^{\infty}}+\left\|A_{1}\right\|_{L^{\infty}}+M_{0} \operatorname{ta}(t)+M_{\infty}$ e $t^{2} a(t)$. The formula (3.11) yields

$$
\begin{aligned}
\left\|\partial_{x} \tilde{A}(t)\right\|_{L^{\infty}} & \leq c(t)+2 M_{\infty} t a(t)^{2} \int_{0}^{t}\left\|\partial_{x} A(\tau)\right\|_{L^{\infty}} d \tau \\
& \leq c(t)\left(1+2 M_{\infty} t a(t)^{2} \int_{0}^{t} \exp \left(2 M_{\infty} \tau^{2} a(\tau)^{2}\right) d \tau\right) \\
& \leq c(t)\left(1+2 M_{\infty} t a(t)^{2} \int_{0}^{t} \exp \left(2 M_{\infty} t a(t)^{2} \tau\right) d \tau\right) \\
& =a_{1}(t) .
\end{aligned}
$$

Similarly we have $\left\|\partial_{t} \tilde{A}(t)\right\|_{L^{\infty}} \leq a_{1}(t), t \in[0, T]$, and from (3.7) one also gets

$$
\left\|\partial_{x} \tilde{E}\right\|_{L^{\infty}(] 0, T[\times \mathbb{R})} \leq\left\|\rho_{\text {ext }}\right\|_{L^{\infty}}+M_{0}+2 M_{\infty} e T+2 M_{\infty} \int_{0}^{T} a(s) a_{1}(s) d s=e_{1} .
$$


For using iterative procedure it is convenient to restrict the domain $D_{T}$ to the set (still denoted $D_{T}$ )

$$
\begin{aligned}
D_{T}=\left\{(E, A) \in L^{\infty}(] 0, T\left[; W^{1, \infty}(\mathbb{R})\right) \times L^{\infty}(] 0, T\left[; W^{2, \infty}(\mathbb{R})\right):\|E\|_{L^{\infty}} \leq e,\left\|\partial_{x} E\right\|_{L^{\infty}} \leq e_{1}\right. \\
\left.\|A(t)\|_{L^{\infty}} \leq a(t), \max \left\{\left\|\partial_{x} A(t)\right\|_{L^{\infty}},\left\|\partial_{t} A(t)\right\|_{L^{\infty}}\right\} \leq a_{1}(t), t \in[0, T]\right\} .
\end{aligned}
$$

For further computations we also need to estimate the $L^{\infty}$ norm of the second space derivative $\partial_{x}^{2} \tilde{A}$. This type of estimate has been obtained in [5] locally in time for the NR case and globally in time for the QR case. We will show that this is possible globally in time in the FR case. We need the following easy lemmas.

Lemma 3.4. Assume that $f_{0}$ is nonnegative satisfying

$$
\left(\tilde{H}_{k}\right) \quad \int_{\mathbb{R}} \int_{\mathbb{R}}\left(1+|p|^{k}\right) f_{0}(x, p) d p d x<+\infty .
$$

Then there is a constant $C$ such that for all regular fields $(E, A) \in D_{T}$ we have

$$
\int_{\mathbb{R}} \int_{\mathbb{R}}|p|^{k} f_{E, A}(t, x, p) d p d x \leq C \int_{\mathbb{R}} \int_{\mathbb{R}}\left(1+|p|^{k}\right) f_{0}(x, p) d p d x, \quad t \in[0, T] .
$$

Proof. For any $t \in[0, T]$ we can write

$$
\int_{\mathbb{R}} \int_{\mathbb{R}}|p|^{k} f_{E, A}(t, x, p) d p d x=\int_{\mathbb{R}} \int_{\mathbb{R}}|p|^{k} f_{0}(X(0 ; t, x, p), P(0 ; t, x, p)) d p d x,
$$

where $(X, P)$ are the characteristics associated to $(E, A)$. Taking into account that

$$
\begin{aligned}
|p-P(0 ; t, x, p)| & \leq R(t)=\int_{0}^{t}\left\{\|E(s)\|_{L^{\infty}}+\left\|A(s) \partial_{x} A(s)\right\|_{L^{\infty}}\right\} d s \\
& \leq T\left(e+a(T) a_{1}(T)\right)=: R, \quad t \in[0, T],
\end{aligned}
$$

we deduce that $|p|^{k} \leq C\left(1+|P(0 ; t, x, p)|^{k}\right)$ and the conclusion follows easily since

$$
\begin{aligned}
\int_{\mathbb{R}} \int_{\mathbb{R}}|p|^{k} f_{0}(X(0), P(0)) d p d x & \leq C \int_{\mathbb{R}} \int_{\mathbb{R}}\left(1+|P(0)|^{k}\right) f_{0}(X(0), P(0)) d p d x \\
& =C \int_{\mathbb{R}} \int_{\mathbb{R}}\left(1+|p|^{k}\right) f_{0}(x, p) d p d x, t \in[0, T] .
\end{aligned}
$$

Lemma 3.5. Assume that the hypotheses of Proposition 3.1 hold and suppose that

$$
M_{k}:=\int_{\mathbb{R}}|p|^{k} n_{0}(p) d p<+\infty .
$$

Then for any $(E, A) \in D_{T}$ we have

$$
\left\|\int_{\mathbb{R}}|p|^{k} f_{E, A}(\cdot, \cdot, p) d p\right\|_{L^{\infty}(] 0, T[\times \mathbb{R})} \leq C\left(M_{0}+M_{k}+M_{\infty}\right),
$$

for a constant $C$ depending on $T$ and the initial conditions. 
Proof. We have for $(t, x) \in[0, T] \times \mathbb{R}$

$$
\begin{aligned}
\int_{\mathbb{R}}|p|^{k} f_{E, A}(t, x, p) d p & =\int_{\mathbb{R}}|p|^{k} f_{0}(X(0 ; t, x, p), P(0 ; t, x, p)) d p \\
& \leq \int_{\mathbb{R}}|p|^{k} n_{0}(P(0 ; t, x, p)) d p \\
& \leq \int_{\mathbb{R}}|p|^{k} n_{0}^{R(t)}(p) d p
\end{aligned}
$$

and the conclusion follows easily since $R(t) \leq t\left(e+a(t) a_{1}(t)\right), \forall t \in[0, T]$.

Proposition 3.6. Assume that the hypotheses of Proposition 3.1 hold. Then for any regular fields $(E, A) \in L^{\infty}(] 0, T\left[; W^{1, \infty}(\mathbb{R})\right) \times L^{\infty}(] 0, T\left[; W^{2, \infty}(\mathbb{R})\right)$ we have the estimate $\left\|\partial_{t} \tilde{E}(t)\right\|_{L^{\infty}} \leq M_{0}+2 M_{\infty} R(t)$ where $R(t)=\int_{0}^{t}\left\{\|E(s)\|_{L^{\infty}}+\left\|A(s) \partial_{x} A(s)\right\|_{L^{\infty}}\right\} d s$, $t \in[0, T]$.

Proof. Observe that under the above hypotheses $j_{E, A}=\int_{\mathbb{R}} \frac{p}{\gamma_{1}} f_{E, A} d p$ is well defined. We prove that $\partial_{t} \tilde{E}=j_{E, A}$. For this pick a function $\varphi \in C_{c}^{1}(] 0, T[\times \mathbb{R})$ and calculate

$$
\begin{aligned}
\int_{0}^{T} \int_{\mathbb{R}} \tilde{E}(t, x) \partial_{t} \varphi d x d t & =\int_{0}^{T} \int_{\mathbb{R}} \int_{\mathbb{R}} f_{0}\left\{\frac{d}{d t} \int_{x}^{X(t ; 0, x, p)} \varphi(t, u) d u-\varphi(t, X(t)) \frac{P(t)}{\gamma_{1}(t)}\right\} d p d x d t \\
& =-\int_{\mathbb{R}} \int_{\mathbb{R}} f_{0}(x, p) \int_{0}^{T} \varphi(t, X(t ; 0, x, p)) \frac{P(t ; 0, x, p)}{\gamma_{1}(t)} d t d p d x \\
& =-\int_{0}^{T} \int_{\mathbb{R}} j_{E, A}(t, x) \varphi(t, x) d x d t
\end{aligned}
$$

which implies that $\partial_{t} \tilde{E}=j_{E, A}$. Therefore we obtain

$$
\left\|\partial_{t} \tilde{E}(t)\right\|_{L^{\infty}} \leq\left\|\rho_{E, A}\right\|_{L^{\infty}} \leq M_{0}+2 M_{\infty} R(t) .
$$

Proposition 3.7. Assume that the hypotheses of Propositions 3.1, 3.2 hold, $A_{0} \in$ $W^{2, \infty}(\mathbb{R}), A_{1} \in W^{1, \infty}(\mathbb{R})$. Moreover we suppose that $M_{1}=\int_{\mathbb{R}}|p| n_{0}(p) d p<+\infty$. Then for any $T>0$ there is a constant $C$ depending on $T$ and the initial conditions such that

$$
\max \left\{\left\|\partial_{x t}^{2} \tilde{A}\right\|_{L^{\infty}(] 0, T[\times \mathbb{R})},\left\|\partial_{x}^{2} \tilde{A}\right\|_{L^{\infty}(] 0, T[\times \mathbb{R})},\left\|\partial_{t}^{2} \tilde{A}\right\|_{L^{\infty}(] 0, T[\times \mathbb{R})}\right\} \leq C, \quad \forall(E, A) \in D_{T} .
$$

Proof. Recall that the first space derivative of $\tilde{A}$ is given by

$$
\partial_{x} \tilde{A}(t, x)=D^{0}(t, x)+\frac{1}{2} D^{-}(t, x)-\frac{1}{2} D^{+}(t, x),
$$

where $D^{0}(t, x)=\frac{1}{2}\left\{A_{0}^{\prime}(x+t)+A_{0}^{\prime}(x-t)\right\}+\frac{1}{2}\left\{A_{1}(x+t)-A_{1}(x-t)\right\}$ and $D^{ \pm}(t, x)=$ $\int_{0}^{t}\left(\rho_{\gamma_{2}} A\right)(s, x \pm(t-s)) d s$. Obviously we have $\left\|\partial_{x} D^{0}\right\|_{L^{\infty}(] 0, T[\times \mathbb{R})} \leq\left\|A_{0}^{\prime \prime}\right\|_{L^{\infty}}+\left\|A_{1}^{\prime}\right\|_{L^{\infty}}$, and it remains to estimate the space derivatives of $D^{ \pm}(t, \cdot)$ for $t \in[0, T]$. Pick a test 
function $\varphi \in C_{c}^{1}(\mathbb{R})$ and by using (2.6) one gets

$$
\begin{aligned}
\int_{\mathbb{R}} D^{ \pm}(t, x) \varphi^{\prime}(x) d x & =\int_{0}^{t} \int_{\mathbb{R}}\left(\rho_{\gamma_{2}} A\right)(s, x \pm(t-s)) \varphi^{\prime}(x) d x d s \\
& =\int_{0}^{t} \int_{\mathbb{R}}\left(\rho_{\gamma_{2}} A\right)(s, x) \varphi^{\prime}(x \mp(t-s)) d x d s \\
& =\int_{0}^{t} \int_{\mathbb{R}} \int_{\mathbb{R}} \frac{f(s, x, p)}{\gamma_{2}} A(s, x) \varphi^{\prime}(x \mp(t-s)) d p d x d s \\
& =\int_{\mathbb{R}} \int_{\mathbb{R}} f_{0}(x, p) I^{ \pm}(t, x, p) d p d x
\end{aligned}
$$

where $I^{ \pm}(t, x, p)=\int_{0}^{t} \frac{A(s, X(s ; 0, x, p))}{\gamma_{2}(s)} \varphi^{\prime}(X(s ; 0, x, p) \mp(t-s)) d s, \forall(t, x, p) \in[0, T] \times \mathbb{R}^{2}$, $\gamma_{2}(s)=\left(1+|P(s ; 0, x, p)|^{2}+|A(s, X(s ; 0, x, p))|^{2}\right)^{1 / 2}$. We need to evaluate $I^{ \pm}(t, x, p)$. The crucial point here is that the velocities of any characteristic (2.4) remain below the characteristic's speed of (1.13)

$$
\left|\frac{d X}{d s}\right|=\left|\frac{P(s ; 0, x, p)}{\gamma_{1}(s)}\right|<1, \quad \forall(s, x, p) \in[0, T] \times \mathbb{R}^{2},
$$

where $\gamma_{1}(s)=\left(1+|P(s ; 0, x, p)|^{2}+|A(s, X(s ; 0, x, p))|^{2}\right)^{1 / 2}$. This fact was also one of the key points of the proofs in [5], 9], 10]. Therefore the following computations are valid:

$$
\begin{aligned}
I^{ \pm}(t, x, p) & =\int_{0}^{t} \frac{A(s, X(s))}{\gamma_{2}(s)\left(X^{\prime}(s) \pm 1\right)} \frac{d}{d s} \varphi(X(s) \mp(t-s)) d s \\
& =\left.\frac{A(s, X(s))}{\gamma_{2}(s)\left(X^{\prime}(s) \pm 1\right)} \varphi(X(s) \mp(t-s))\right|_{\substack{s=t \\
s=0}} ^{t} \\
& -\int_{0}^{t} \frac{d}{d s}\left\{\frac{A(s, X(s))}{\gamma_{2}(s)\left(X^{\prime}(s) \pm 1\right)}\right\} \varphi(X(s) \mp(t-s)) d s \\
& =I_{1} \varphi(X(t))-I_{2} \varphi(x \mp t)-\int_{0}^{t} I_{3}(s) \varphi(X(s) \mp(t-s)) d s .
\end{aligned}
$$

Note that we have

$$
\begin{aligned}
\frac{1}{\left|\gamma_{2}(s)\left(X^{\prime}(s) \pm 1\right)\right|} & =\frac{\sqrt{1+|P(s)|^{2}+|A(s, X(s))|^{2}} \mp P(s)}{1+|A(s, X(s))|^{2}} \\
& \leq 2 \frac{\sqrt{1+|P(s)|^{2}+|A(s, X(s))|^{2}}}{1+|A(s, X(s))|^{2}} \\
& \leq 2(1+|P(s)|) .
\end{aligned}
$$

With the previous notations we obtain

$$
\begin{aligned}
\int_{\mathbb{R}} D^{ \pm}(t, x) \varphi^{\prime}(x) d x & =\int_{\mathbb{R}} \int_{\mathbb{R}} f_{0}(x, p) I_{1} \varphi(X(t)) d p d x-\int_{\mathbb{R}} \int_{\mathbb{R}} f_{0}(x, p) I_{2} \varphi(x \mp t) d p d x \\
& -\int_{\mathbb{R}} \int_{\mathbb{R}} f_{0}(x, p) \int_{0}^{t} I_{3}(s) \varphi(X(s) \mp(t-s)) d s d p d x \\
& =Q_{1}+Q_{2}+Q_{3} .
\end{aligned}
$$


Estimate of $Q_{1}$

$$
\begin{aligned}
\left|Q_{1}\right| & \leq \int_{\mathbb{R}} \int_{\mathbb{R}} f_{0}(x, p) \frac{|A(t, X(t))|}{\left|\gamma_{2}(t)\left(X^{\prime}(t) \pm 1\right)\right|}|\varphi(X(t))| d p d x \\
& \leq 2 a(t) \int_{\mathbb{R}} \int_{\mathbb{R}} f_{0}(x, p)(1+|P(t)|)|\varphi(X(t))| d p d x \\
& =2 a(t) \int_{\mathbb{R}} \int_{\mathbb{R}} f_{E, A}(t, X(t), P(t))(1+|P(t)|)|\varphi(X(t))| d p d x \\
& =2 a(t) \int_{\mathbb{R}} \int_{\mathbb{R}} f_{E, A}(t, x, p)(1+|p|)|\varphi(x)| d p d x \\
& \leq 2 a(t)\left(\left\|\int_{\mathbb{R}} f_{E, A}(t, \cdot, p) d p\right\|_{L^{\infty}}+\left\|\int_{\mathbb{R}} f_{E, A}(t, \cdot, p)|p| d p\right\|_{L^{\infty}}\right)\|\varphi\|_{L^{1}} \\
& \leq C_{1}\|\varphi\|_{L^{1}},
\end{aligned}
$$

where $C_{1}=2 a(T)\left(M_{0}+2 M_{\infty} T\left(e+a(T) a_{1}(T)\right)+C\left(M_{0}+M_{1}+M_{\infty}\right)\right)$.

Estimate of $Q_{2}$

$$
\begin{aligned}
\left|Q_{2}\right| & \leq \int_{\mathbb{R}} \int_{\mathbb{R}} f_{0}(x, p) \frac{|A(0, x)|}{\left|\gamma_{2}(0)\left(X^{\prime}(0) \pm 1\right)\right|}|\varphi(x \mp t)| d p d x \\
& \leq 2\left\|A_{0}\right\|_{L^{\infty}} \int_{\mathbb{R}} \int_{\mathbb{R}} f_{0}(x, p)(1+|p|)|\varphi(x \mp t)| d p d x \\
& \leq 2\left\|A_{0}\right\|_{L^{\infty}}\left(\left\|\int_{\mathbb{R}} f_{0}(\cdot, p) d p\right\|_{L^{\infty}}+\left\|\int_{\mathbb{R}} f_{0}(\cdot, p)|p| d p\right\|_{L^{\infty}}\right)\|\varphi\|_{L^{1}} \\
& \leq C_{2}\|\varphi\|_{L^{1}},
\end{aligned}
$$

with $C_{2}=2\left\|A_{0}\right\|_{L^{\infty}}\left(M_{0}+M_{1}\right)$.

Estimate of $Q_{3}$

We obtain

$$
\begin{aligned}
\left|I_{3}(s)\right| & =\left|\frac{d}{d s}\left\{\frac{A(s, X(s))}{P(s) \pm \sqrt{1+|P(s)|^{2}+|A(s, X(s))|^{2}}}\right\}\right| \\
& =\left|\frac{d}{d s}\left\{\frac{A(s, X(s))}{1+|A(s, X(s))|^{2}}\left(\sqrt{1+|P(s)|^{2}+|A(s, X(s))|^{2}} \mp P(s)\right)\right\}\right| \\
& \leq C(1+|P(s)|) .
\end{aligned}
$$

Therefore we have

$$
\begin{aligned}
\left|Q_{3}\right| & \leq \int_{\mathbb{R}} \int_{\mathbb{R}} f_{0}(x, p) \int_{0}^{t} C(1+|P(s)|)|\varphi(X(s) \mp(t-s))| d s d p d x \\
& =C \int_{0}^{t} \int_{\mathbb{R}} \int_{\mathbb{R}} f_{E, A}(s, x, p)(1+|p|)|\varphi(x \mp(t-s))| d p d x d s \\
& \leq C \int_{0}^{t} \int_{\mathbb{R}}|\varphi(x \mp(t-s))|\left\{\left\|\int_{\mathbb{R}} f_{E, A}(s, \cdot, p) d p\right\|_{\infty}+\left\|\int_{\mathbb{R}} f_{E, A}(s, \cdot, p)|p| d p\right\|_{\infty}\right\} d x \\
& \leq C_{3}\|\varphi\|_{L^{1}} .
\end{aligned}
$$


The equality (3.19) and the inequalities (3.20), (3.21), (3.22) imply

$$
\left|\int_{\mathbb{R}} D^{ \pm}(t, x) \varphi^{\prime}(x) d x\right| \leq\left(C_{1}+C_{2}+C_{3}\right)\|\varphi\|_{L^{1}},
$$

and therefore $\partial_{x} D^{ \pm} \in L^{\infty}(] 0, T[\times \mathbb{R}),\left\|\partial_{x} D^{ \pm}\right\|_{L^{\infty}} \leq C_{1}+C_{2}+C_{3}=: C_{4}$. We finally obtain that $\left\|\partial_{x}^{2} \tilde{A}\right\|_{L^{\infty}} \leq\left\|A_{0}^{\prime \prime}\right\|_{L^{\infty}}+\left\|A_{1}^{\prime}\right\|_{L^{\infty}}+C_{4}=: C_{5}, \quad \forall(E, A) \in D_{T}$. The second derivative $\partial_{x t}^{2} \tilde{A}$ satisfies the same estimate since we have

$\partial_{t} \tilde{A}(t, x)=\frac{1}{2}\left\{A_{0}^{\prime}(x+t)-A_{0}^{\prime}(x-t)\right\}+\frac{1}{2}\left\{A_{1}(x+t)+A_{1}(x-t)\right\}-\frac{1}{2} D^{+}(t, x)-\frac{1}{2} D^{-}(t, x)$,

and therefore

$$
\begin{aligned}
\left\|\partial_{x t}^{2} \tilde{A}\right\|_{L^{\infty}} & \leq\left\|A_{0}^{\prime \prime}\right\|_{L^{\infty}}+\left\|A_{1}^{\prime}\right\|_{L^{\infty}}+\frac{1}{2}\left(\left\|\partial_{x} D^{+}\right\|_{L^{\infty}}+\left\|\partial_{x} D^{-}\right\|_{L^{\infty}}\right) \\
& \leq\left\|A_{0}^{\prime \prime}\right\|_{L^{\infty}}+\left\|A_{1}^{\prime}\right\|_{L^{\infty}}+C_{4} \\
& =C_{5} .
\end{aligned}
$$

By using the wave equation (1.13), we also obtain an estimate for $\partial_{t}^{2} \tilde{A}$ :

$$
\begin{aligned}
\left\|\partial_{t}^{2} \tilde{A}(t)\right\|_{L^{\infty}} & \leq\left\|\partial_{x}^{2} \tilde{A}(t)\right\|_{L^{\infty}}+\left\|\rho_{\gamma_{2}}(t)\right\|_{L^{\infty}}\|A(t)\|_{L^{\infty}} \\
& \leq C_{5}+\left\{M_{0}+2 M_{\infty} T\left(e+a(T) a_{1}(T)\right)\right\} a(T)=: C_{6} .
\end{aligned}
$$

We restrict one more time the domain $D_{T}$ to the set (still denoted $D_{T}$ )

$$
\begin{aligned}
D_{T}= & \left\{(E, A) \in W^{1, \infty}(] 0, T[\times \mathbb{R}) \times W^{2, \infty}(] 0, T[\times \mathbb{R}) \mid\|E\|_{L^{\infty}} \leq e,\left\|\partial_{x} E\right\|_{L^{\infty}} \leq e_{1},\right. \\
& \left\|\partial_{t} E\right\|_{L^{\infty}} \leq e_{2}, \max \left\{\left\|\partial_{x}^{2} A\right\|_{L^{\infty}},\left\|\partial_{x t}^{2} A\right\|_{L^{\infty}}\right\} \leq C_{5},\left\|\partial_{t}^{2} A\right\|_{L^{\infty}} \leq C_{6}, \\
& \left.\|A(t)\|_{L^{\infty}} \leq a(t), \max \left\{\left\|\partial_{x} A(t)\right\|_{L^{\infty}},\left\|\partial_{t} A(t)\right\|_{L^{\infty}}\right\} \leq a_{1}(t), t \in[0, T]\right\}
\end{aligned}
$$

where $e_{2}=M_{0}+2 M_{\infty} T\left(e+a(T) a_{1}(T)\right)$. Observe that this set is left invariant by $\mathcal{F}$.

3.3. Estimates for $\mathcal{F}\left(E_{1}, A_{1}\right)-\mathcal{F}\left(E_{2}, A_{2}\right)$. Note that the domain $D_{T}$ constructed above is a closed bounded set of $X_{T}=W^{1, \infty}(] 0, T[\times \mathbb{R}) \times W^{2, \infty}(] 0, T[\times \mathbb{R})$. Our goal now is to evaluate the difference $\mathcal{F}\left(E_{1}, A_{1}\right)-\mathcal{F}\left(E_{2}, A_{2}\right)$ in terms of $\left(E_{1}-E_{2}, A_{1}-A_{2}\right)$ when $\left(E_{k}, A_{k}\right)_{k \in\{1,2\}}$ belong to $D_{T}$. We suppose that $f_{0}$ is nonnegative, belongs to $L^{1}\left(\mathbb{R}^{2}\right)$ and satisfies $(H),\left(H_{0}\right),\left(\tilde{H}_{1}\right),\left(H_{\infty}\right), \rho_{\text {ext }}$ is nonnegative, $\rho_{\text {ext }} \in L^{1}(\mathbb{R}) \cap L^{\infty}(\mathbb{R})$, $E_{0}^{\prime}=\rho_{\text {ext }}-\int_{\mathbb{R}} f_{0}(\cdot, p) d p, A_{0} \in W^{2, \infty}(\mathbb{R})$, and $A_{1} \in W^{1, \infty}(\mathbb{R})$. We use the notation $\|\mid(E, A)(t)\|\|=\| E(t)\left\|_{L^{\infty}}+\right\| A(t)\left\|_{L^{\infty}}+\right\| \partial_{x} A(t)\left\|_{L^{\infty}}+\right\| \partial_{t} A(t) \|_{L^{\infty}}$.

Estimate for $\tilde{E}_{1}-\tilde{E}_{2}$

Consider a test function $\varphi \in L^{1}(\mathbb{R})$. From the definitions of $\tilde{E}_{1}, \tilde{E}_{2}$ one gets

$$
\begin{aligned}
Q_{4} & :=\left|\int_{\mathbb{R}}\left(\tilde{E}_{1}(t, x)-\tilde{E}_{2}(t, x)\right) \varphi(x) d x\right| \leq \int_{\mathbb{R}} \int_{\mathbb{R}} f_{0}(x, p)\left|\int_{X_{2}(t ; 0, x, p)}^{X_{1}(t ; 0, x, p)}\right| \varphi(u)|d u| d p d x \\
& \leq \int_{\mathbb{R}} \int_{\mathbb{R}} f_{0}(x, p)|\varphi(u)| \mathbf{1}_{\left\{\left|u-X_{1}(t)\right| \leq\left|X_{2}(t)-X_{1}(t)\right|\right\}} d u d p d x
\end{aligned}
$$


where $\left(X_{k}, P_{k}\right)_{k \in\{1,2\}}$ are the characteristics associated with the fields $\left(E_{k}, A_{k}\right)_{k \in\{1,2\}}$. By Proposition 2.3 we deduce that

$$
\begin{aligned}
\left|X_{1}(t ; 0, x, p)-X_{2}(t ; 0, x, p)\right| & +\left|P_{1}(t ; 0, x, p)-P_{2}(t ; 0, x, p)\right| \\
& \leq C \int_{0}^{t}\left\|\left|\left(E_{1}-E_{2}, A_{1}-A_{2}\right)(s)\right|\right\| d s=: \delta(t),
\end{aligned}
$$

and therefore we can write

$$
\begin{aligned}
Q_{4} & \leq \int_{\mathbb{R}}|\varphi(u)| \int_{\mathbb{R}} \int_{\mathbb{R}} f_{0}(x, p) \mathbf{1}_{\left\{\left|u-X_{1}(t)\right| \leq \delta(t)\right\}} d p d x d u \\
& =\int_{\mathbb{R}}|\varphi(u)| \int_{\mathbb{R}} \int_{\mathbb{R}} f_{E_{1}, A_{1}}(t, x, p) \mathbf{1}_{\{|u-x| \leq \delta(t)\}} d p d x d u \\
& =\int_{\mathbb{R}}|\varphi(u)| \int_{\mathbb{R}} \rho_{E_{1}, A_{1}}(t, x) \mathbf{1}_{\{|u-x| \leq \delta(t)\}} d x d u \\
& \leq 2 \delta(t)\left\|\rho_{E_{1}, A_{1}}(t)\right\|_{L^{\infty}}\|\varphi\|_{L^{1}}, \quad \forall \varphi \in L^{1}(\mathbb{R}) .
\end{aligned}
$$

We deduce that for any $T>0$ there is a constant depending on $T$ and the initial conditions such that for $\left(E_{k}, A_{k}\right) \in D_{T}, k \in\{1,2\}$, we have

$$
\left\|\left(\tilde{E}_{1}-\tilde{E}_{2}\right)(t)\right\|_{L^{\infty}} \leq C \int_{0}^{t}\left\|\left(E_{1}-E_{2}, A_{1}-A_{2}\right)(s)\right\| d s, \quad \forall t \in[0, T] .
$$

REMARK 3.8. We also retain the inequality

$$
\int_{\mathbb{R}} \int_{\mathbb{R}} f_{0}(x, p)\left|\int_{X_{2}(t ; 0, x, p)}^{X_{1}(t ; 0, x, p)} \varphi(u) d u\right| d p d x \leq C\|\varphi\|_{L^{1}} \int_{0}^{t}\|\|\left(E_{1}-E_{2}, A_{1}-A_{2}\right)(s)\|\| d s,
$$

for all $t \in[0, T]$.

Estimate for $\tilde{A}_{1}-\tilde{A}_{2}$

For any test function $\varphi \in L^{1}(\mathbb{R})$ we have

$$
\begin{aligned}
Q_{5} & :=\left|\int_{\mathbb{R}}\left(\tilde{A}_{1}(t, x)-\tilde{A}_{2}(t, x)\right) \varphi(x) d x\right| \\
& =\frac{1}{2}\left|\int_{\mathbb{R}} \int_{0}^{t} \int_{x-(t-s)}^{x+(t-s)}\left(\rho_{1, \gamma_{2}} A_{1}-\rho_{2, \gamma_{2}} A_{2}\right)(s, y) d y d s \varphi(x) d x\right| \\
& \leq \frac{1}{2}\left|\int_{\mathbb{R}} \int_{0}^{t} \int_{x-(t-s)}^{x+(t-s)}\left\{\rho_{1, \gamma_{2}}\left(A_{1}-A_{2}\right)\right\}(s, y) d y d s \varphi(x) d x\right| \\
& \left.+\frac{1}{2} \mid \int_{\mathbb{R}} \int_{0}^{t} \int_{x-(t-s)}^{x+(t-s)}\left\{\left(\rho_{1, \gamma_{2}}-\rho_{2, \gamma_{2}}\right) A_{2}\right)\right\}(s, y) d y d s \varphi(x) d x \mid \\
& =\frac{1}{2}\left(\left|I_{1}\right|+\left|I_{2}\right|\right) .
\end{aligned}
$$

We easily check that

$$
\left|I_{1}\right| \leq \int_{0}^{t}\left\|A_{1}(s)-A_{2}(s)\right\|_{L^{\infty}(\mathbb{R})} d s\left\|f_{0}\right\|_{L^{1}\left(\mathbb{R}^{2}\right)}\|\varphi\|_{L^{1}(\mathbb{R})} .
$$


For analyzing the term $I_{2}$ we introduce the notation $\psi(t, s, y)=\int_{y-(t-s)}^{y+(t-s)} \varphi(x) d x$. We have

$$
\begin{aligned}
\left|I_{2}\right| & =\left|\int_{0}^{t} \int_{\mathbb{R}}\left\{\left(\rho_{1, \gamma_{2}}-\rho_{2, \gamma_{2}}\right) A_{2}\right\}(s, y) \psi(t, s, y) d y d s\right| \\
& =\left|\int_{0}^{t} \int_{\mathbb{R}} \int_{\mathbb{R}}\left[\frac{f_{E_{1}, A_{1}}(s, y, p)}{\sqrt{1+|p|^{2}+\left|A_{1}(s, y)\right|^{2}}}-\frac{f_{E_{2}, A_{2}}(s, y, p)}{\sqrt{1+|p|^{2}+\left|A_{2}(s, y)\right|^{2}}}\right] A_{2} \psi d p d y d s\right| \\
& =\left|\int_{\mathbb{R}} \int_{\mathbb{R}} f_{0}(x, p) \int_{0}^{t} \sum_{k=1}^{2}(-1)^{k} \frac{A_{2}\left(s, X_{k}(s)\right) \psi\left(t, s, X_{k}(s)\right)}{\sqrt{1+\left|P_{k}(s)\right|^{2}+\left|A_{k}\left(s, X_{k}(s)\right)\right|^{2}}} d s d p d x\right| \\
& \leq \int_{0}^{t} \int_{\mathbb{R}} \int_{\mathbb{R}} f_{0}(x, p)\left|I_{3}(s, x, p)\right| d p d x d s,
\end{aligned}
$$

where

$$
I_{3}(s, x, p)=\sum_{k=1}^{2}(-1)^{k} \frac{A_{2}\left(s, X_{k}(s)\right) \psi\left(t, s, X_{k}(s)\right)}{\sqrt{1+\left|P_{k}(s)\right|^{2}+\mid A_{k}\left(s,\left.X_{k}(s)\right|^{2}\right.}} .
$$

Observe that

$$
\begin{aligned}
\left|I_{3}(s, x, p)\right| & \leq\left|A_{2}\left(s, X_{2}(s)\right)-A_{2}\left(s, X_{1}(s)\right)\right|\left|\psi\left(t, s, X_{2}(s)\right)\right| \\
& +\left|A_{2}\left(s, X_{1}(s)\right)\right|\left|\psi\left(t, s, X_{2}(s)\right)-\psi\left(t, s, X_{1}(s)\right)\right| \\
& +\frac{\left|\psi\left(t, s, X_{2}(s)\right)\right|\left\{\left|P_{1}(s)-P_{2}(s)\right|+\left|A_{1}\left(s, X_{1}(s)\right)-A_{2}\left(s, X_{2}(s)\right)\right|\right\}}{\sqrt{1+\left|P_{1}(s)\right|^{2}+\left|A_{1}\left(s, X_{1}(s)\right)\right|^{2}}} \\
& \leq 2\left|A_{2}\left(s, X_{2}(s)\right)-A_{2}\left(s, X_{1}(s)\right)\right|\left|\psi\left(t, s, X_{2}(s)\right)\right| \\
& +\left|A_{2}\left(s, X_{1}(s)\right)\right|\left|\psi\left(t, s, X_{2}(s)\right)-\psi\left(t, s, X_{1}(s)\right)\right| \\
& +\left\|A_{1}(s)-A_{2}(s)\right\|_{L^{\infty}}\left|\psi\left(t, s, X_{2}(s)\right)\right|+\left|P_{1}(s)-P_{2}(s)\right|\left|\psi\left(t, s, X_{2}(s)\right)\right| \\
& \leq 2\left\|\partial_{x} A_{2}(s)\right\|_{L^{\infty}}\left|X_{1}(s)-X_{2}(s)\right|\|\varphi\|_{L^{1}} \\
& +\left|P_{1}(s)-P_{2}(s)\right|\|\varphi\|_{L^{1}}+\left\|A_{1}(s)-A_{2}(s)\right\|_{L^{\infty}}\|\varphi\|_{L^{1}} \\
& +\left\|A_{2}(s)\right\|_{L^{\infty}}\left\{\left|\int_{X_{2}(s)+(t-s)}^{X_{1}(s)+(t-s)} \varphi(u) d u\right|+\left|\int_{X_{2}(s)-(t-s)}^{X_{1}(s)-(t-s)} \varphi(u) d u\right|\right\} .
\end{aligned}
$$

Combining (3.28), (3.29) and using Remark 3.8 yields

$$
\left|I_{2}\right| \leq C \int_{0}^{t}\|\|\left(E_{1}-E_{2}, A_{1}-A_{2}\right)(s)\|\| d s\|\varphi\|_{L^{1}} .
$$

Finally (3.26), (3.27) and (3.30) imply that for all $t \in[0, T],\left(E_{1}, A_{1}\right),\left(E_{2}, A_{2}\right) \in D_{T}$ we have

$$
\left\|\left(\tilde{A}_{1}-\tilde{A}_{2}\right)(t)\right\|_{L^{\infty}(\mathbb{R})} \leq C \int_{0}^{t}\left\|\mid\left(E_{1}-E_{2}, A_{1}-A_{2}\right)(s)\right\| \| d s,
$$

for some constant depending on $T$ and the initial conditions. 
Estimate for $\partial_{x} \tilde{A}_{1}-\partial_{x} \tilde{A}_{2}$

For any test function $\varphi \in C_{c}^{0}(\mathbb{R})$ we need to estimate

$$
\begin{aligned}
Q_{6} & :=\int_{\mathbb{R}}\left(\partial_{x} \tilde{A}_{1}-\partial_{x} \tilde{A}_{2}\right)(t, x) \varphi(x) d x \\
& =-\frac{1}{2} \int_{0}^{t} \int_{\mathbb{R}}\left(\rho_{1, \gamma_{2}} A_{1}-\rho_{2, \gamma_{2}} A_{2}\right)(s, x) \varphi(x-t+s) d x d s \\
& +\frac{1}{2} \int_{0}^{t} \int_{\mathbb{R}}\left(\rho_{1, \gamma_{2}} A_{1}-\rho_{2, \gamma_{2}} A_{2}\right)(s, x) \varphi(x+t-s) d x d s \\
& =-\frac{1}{2} Q_{6}^{+}+\frac{1}{2} Q_{6}^{-} .
\end{aligned}
$$

We have

$$
\begin{aligned}
Q_{6}^{ \pm} & =\sum_{k=1}^{2}(-1)^{k-1} \int_{0}^{t} \int_{\mathbb{R}} \int_{\mathbb{R}} \frac{f_{E_{k}, A_{k}}(s, x, p)}{\gamma_{2}} A_{k}(s, x) \varphi(x \mp(t-s)) d p d x d s \\
& =\sum_{k=1}^{2}(-1)^{k-1} \int_{\mathbb{R}} \int_{\mathbb{R}} f_{0} \int_{0}^{t} \frac{A_{k}\left(s, X_{k}(s)\right)}{P_{k}(s) \pm \gamma_{1}\left(P_{k}(s), A_{k}\left(s, X_{k}(s)\right)\right)} \frac{d}{d s} \int_{0}^{X_{k}(s) \mp(t-s)} \varphi(u) d u d s d p d x \\
& =\sum_{k=1}^{2}(-1)^{k-1} \int_{\mathbb{R}} \int_{\mathbb{R}} f_{0}(x, p) \int_{0}^{t}\left\{G^{ \pm}\left(P_{k}(s), A_{k}\left(s, X_{k}(s)\right)\right) \frac{d}{d s} \int_{0}^{X_{k}(s) \mp(t-s)} \varphi(u) d u\right\} d s d p d x,
\end{aligned}
$$

where $G^{ \pm}(P, A)=\frac{A}{P \pm \gamma_{1}(P, A)}$. Observe that the functions $G^{ \pm}$are well defined since $P+\gamma_{1}(P, A)>0$ and $P-\gamma_{1}(P, A)<0, \forall(P, A) \in \mathbb{R}^{2}$. In order to simplify our further computations we introduce some notations. Consider $G=G(P, A)$ a smooth function $\left(C^{2}\right)$. For any pair of regular fields $(E, A) \in X_{T}$ we construct the derivative of $G$ along the characteristic curves corresponding to $(E, A)$, i.e., for all $(t, x, p) \in[0, T] \times \mathbb{R}^{2}$ we compute

$$
\begin{aligned}
& \lim _{s \rightarrow t} \frac{G(P(s ; t, x, p), A(s, X(s ; t, x, p)))-G(p, A(t, x))}{s-t} \\
= & -\left(E(t, x)+\frac{A(t, x) \partial_{x} A}{\gamma_{2}(p, A(t, x))}\right) \partial_{P} G+\left(\partial_{t} A+\frac{p \partial_{x} A}{\gamma_{1}(p, A(t, x))}\right) \partial_{A} G \\
= & H\left(p, A(t, x), \partial_{x} A(t, x), \partial_{t} A(t, x), E(t, x)\right),
\end{aligned}
$$

with the notation

$$
H(P, A, B, C, E)=-\partial_{P} G(P, A)\left(E+\frac{A B}{\gamma_{2}(P, A)}\right)+\partial_{A} G(P, A)\left(C+\frac{P B}{\gamma_{1}(P, A)}\right),
$$

for all $(P, A, B, C, E) \in \mathbb{R}^{5}$. For $l \in\{1,2\}$ we introduce the class $\mathcal{C}_{l}$ of smooth functions $G \in C^{2}$ such that

$$
\max \left\{|G|,\left|\partial_{P} G\right|,\left|\partial_{A} G\right|\right\}(P, A) \leq g(A)\left(1+|P|^{l}\right), \quad \forall(P, A) \in \mathbb{R}^{2},
$$

for some continuous function $g$ and

$$
\max \left\{|H|,\left|\partial_{P} H\right|,\left|\partial_{A} H\right|,\left|\partial_{B} H\right|,\left|\partial_{C} H\right|,\left|\partial_{E} H\right|\right\}(P, A, B, C, E) \leq h(A, B, C, E)\left(1+|P|^{l}\right),
$$
for all $(P, A, B, C, E) \in \mathbb{R}^{5}$ and some continuous function $h$. 
Lemma 3.9. Assume that $\left(E_{k}, A_{k}\right) \in D_{T}, k \in\{1,2\}, \varphi \in C_{c}^{0}(\mathbb{R})$ and $G \in \mathcal{C}_{1}$. We denote by $\left(X_{k}, P_{k}\right)_{k \in\{1,2\}}$ the characteristics corresponding to $\left(E_{k}, A_{k}\right)_{k \in\{1,2\}}$. Then we have the inequality

$$
\begin{aligned}
& \left|\sum_{k=1}^{2}(-1)^{k} \int_{0}^{t} G\left(P_{k}(s ; 0, x, p), A_{k}\left(s, X_{k}(s ; 0, x, p)\right)\right) \frac{d}{d s}\left(\int_{0}^{X_{k}(s) \mp(t-s)} \varphi(u) d u\right) d s\right| \\
\leq & C\left(1+\left|P_{1}(t)\right|+\left|P_{2}(t)\right|\right)\left(\left\|\left(A_{1}-A_{2}\right)(t)\right\|_{\infty}+\left|\left(X_{1}-X_{2}\right)(t)\right|+\left|\left(P_{1}-P_{2}\right)(t)\right|\right)\|\varphi\|_{L^{1}} \\
+ & C\left(1+\left|P_{2}(t)\right|\right)\left|\int_{X_{2}(t)}^{X_{1}(t)} \varphi(u) d u\right|+\int_{0}^{t}\left\{C\left(1+\left|P_{1}(s)\right|+\left|P_{2}(s)\right|\right)\right. \\
\times & \left(||\left|\left(E_{1}-E_{2}, A_{1}-A_{2}\right)(s)\right|||+\left|X_{1}(s)-X_{2}(s)\right|+\left|P_{1}(s)-P_{2}(s)\right|\right)\|\varphi\|_{L^{1}} \\
+ & \left.C\left(1+\left|P_{2}(s)\right|\right)\left|\int_{X_{2}(s) \mp(t-s)}^{X_{1}(s) \mp(t-s)} \varphi(u) d u\right|\right\} d s,
\end{aligned}
$$

for some constant depending on $T$ and the initial conditions.

Proof. After integration by parts we have for $k \in\{1,2\}$

$$
\int_{0}^{t} G\left(P_{k}(s), A_{k}\left(s, X_{k}(s)\right)\right) \frac{d}{d s}\left(\int_{0}^{X_{k}(s) \mp(t-s)} \varphi(u) d u\right) d s=I_{k}^{t}-I_{k}^{0}-\int_{0}^{t} I_{k}(s) d s,
$$

where $I_{k}^{t}=G\left(P_{k}(t), A_{k}\left(t, X_{k}(t)\right)\right) \int_{0}^{X_{k}(t)} \varphi(u) d u, I_{k}^{0}=G\left(p, A_{k}(0, x)\right) \int_{0}^{x \mp t} \varphi(u) d u$ and

$$
\begin{aligned}
I_{k}(s) & =H\left(P_{k}(s), A_{k}\left(s, X_{k}(s)\right), \partial_{x} A_{k}\left(s, X_{k}(s)\right), \partial_{t} A_{k}\left(s, X_{k}(s)\right), E_{k}\left(s, X_{k}(s)\right)\right) \\
& \times \int_{0}^{X_{k}(s) \mp(t-s)} \varphi(u) d u .
\end{aligned}
$$

Estimate of $I_{1}^{t}-I_{2}^{t}$

$$
\begin{aligned}
\left|I_{1}^{t}-I_{2}^{t}\right| & \leq\left|\sum_{k=1}^{2}(-1)^{k} G\left(P_{k}(t), A_{k}\left(t, X_{k}(t)\right)\right)\right|\left|\int_{0}^{X_{1}(t)} \varphi(u) d u\right| \\
& +\left|G\left(P_{2}(t), A_{2}\left(t, X_{2}(t)\right)\right)\right|\left|\int_{X_{1}(t)}^{X_{2}(t)} \varphi(u) d u\right| .
\end{aligned}
$$

Since $\left(E_{k}, A_{k}\right) \in D_{T}$ we have $\left\|A_{k}(t)\right\|_{L^{\infty}} \leq a(T), k \in\{1,2\}, t \in[0, T]$, and by using the fact that $G \in \mathcal{C}_{1}$ we have

$$
\begin{aligned}
& \left|\sum_{k=1}^{2}(-1)^{k} G\left(P_{k}(t), A_{k}\left(t, X_{k}(t)\right)\right)\right|=\left|\int_{0}^{1} \frac{d}{d \tau} G\left(\left(P_{2}, A_{2}\right)+\tau\left(P_{1}-P_{2}, A_{1}-A_{2}\right)\right) d \tau\right| \\
\leq & \int_{0}^{1} g\left(\tau A_{1}+(1-\tau) A_{2}\right)\left(1+\left|\tau P_{1}+(1-\tau) P_{2}\right|\right) d \tau \\
\times & \left(\left|P_{1}(t)-P_{2}(t)\right|+\left|A_{1}\left(t, X_{1}(t)\right)-A_{2}\left(t, X_{2}(t)\right)\right|\right) \\
\leq & \sup _{|A| \leq a(T)} g(A)\left(1+\left|P_{1}(t)\right|+\left|P_{2}(t)\right|\right)\left(\left|P_{1}(t)-P_{2}(t)\right|+\left|\sum_{k=1}^{2}(-1)^{k} A_{k}\left(t, X_{k}(t)\right)\right|\right) .
\end{aligned}
$$


Since $\left\|\partial_{x} A_{k}\right\|_{L^{\infty}} \leq a_{1}(T), k \in\{1,2\}$, we have

$$
\begin{aligned}
\left|\sum_{k=1}^{2}(-1)^{k} A_{k}\left(t, X_{k}(t)\right)\right| & \leq\left|A_{1}\left(t, X_{1}(t)\right)-A_{1}\left(t, X_{2}(t)\right)\right|+\left|A_{1}\left(t, X_{2}(t)\right)-A_{2}\left(t, X_{2}(t)\right)\right| \\
& \leq a_{1}(T)\left|X_{1}(t)-X_{2}(t)\right|+\left\|A_{1}(t)-A_{2}(t)\right\|_{L^{\infty}}
\end{aligned}
$$

By collecting the inequalities (3.37), (3.38), (3.39) we obtain

$$
\begin{aligned}
\left|I_{1}^{t}-I_{2}^{t}\right| & \leq C\left(1+\left|P_{1}(t)\right|+\left|P_{2}(t)\right|\right)\left(\left|X_{1}(t)-X_{2}(t)\right|+\left|P_{1}(t)-P_{2}(t)\right|\right. \\
& \left.+\left\|A_{1}(t)-A_{2}(t)\right\|_{L^{\infty}}\right)\|\varphi\|_{L^{1}}+C\left(1+\left|P_{2}(t)\right|\right)\left|\int_{X_{2}(t)}^{X_{1}(t)} \varphi(u) d u\right| .
\end{aligned}
$$

Estimate of $I_{1}^{0}-I_{2}^{0}$

The term $I_{1}^{0}-I_{2}^{0}$ vanishes since $A_{1}(0, x)=A_{2}(0, x)=A_{0}(x), \quad x \in \mathbb{R}$,

$$
I_{1}^{0}-I_{2}^{0}=0 \text {. }
$$

Estimate of $I_{1}(s)-I_{2}(s)$

We use the notation

$$
Z_{k}(s)=\left(P_{k}(s), A_{k}\left(s, X_{k}(s)\right), \partial_{x} A_{k}\left(s, X_{k}(s)\right), \partial_{t} A_{k}\left(s, X_{k}(s)\right), E_{k}\left(s, X_{k}(s)\right)\right) \in \mathbb{R}^{5},
$$

for $k \in\{1,2\}$ and $s \in[0, T]$. As before we can write

$$
\begin{aligned}
\left|I_{1}(s)-I_{2}(s)\right| & \leq\left|\left(H\left(Z_{1}(s)\right)-H\left(Z_{2}(s)\right)\right) \int_{0}^{X_{1}(s) \mp(t-s)} \varphi(u) d u\right| \\
& +\left|H\left(Z_{2}(s)\right)\right|\left|\int_{X_{2}(s) \mp(t-s)}^{X_{1}(s) \mp(t-s)} \varphi(u) d u\right|
\end{aligned}
$$

Using the hypothesis $G \in \mathcal{C}_{1}$ yields

$$
\begin{aligned}
\left|H\left(Z_{1}(s)\right)-H\left(Z_{2}(s)\right)\right| & \leq \int_{0}^{1}\left|\nabla H\left(\tau Z_{1}(s)+(1-\tau) Z_{2}(s)\right)\right| d \tau\left|Z_{1}(s)-Z_{2}(s)\right| \\
& \leq C\left(1+\left|P_{1}(s)\right|+\left|P_{2}(s)\right|\right)\left|Z_{1}(s)-Z_{2}(s)\right| .
\end{aligned}
$$

It remains to estimate the difference $\left|Z_{1}(s)-Z_{2}(s)\right|$. We have

$$
\begin{aligned}
\left|Z_{1}(s)-Z_{2}(s)\right| & \leq\left|P_{1}(s)-P_{2}(s)\right|+\left\|\partial_{x} A_{1}(s)\right\|_{\infty}\left|X_{1}(s)-X_{2}(s)\right|+\left\|\left(A_{1}-A_{2}\right)(s)\right\|_{\infty} \\
& +\left\|\partial_{x}^{2} A_{1}(s)\right\|_{L^{\infty}}\left|X_{1}(s)-X_{2}(s)\right|+\left\|\partial_{x}\left(A_{1}-A_{2}\right)(s)\right\|_{L^{\infty}} \\
& +\left\|\partial_{x t}^{2} A_{1}(s)\right\|_{L^{\infty}}\left|X_{1}(s)-X_{2}(s)\right|+\left\|\partial_{t}\left(A_{1}-A_{2}\right)(s)\right\|_{L^{\infty}} \\
& +\left\|\partial_{x} E_{1}(s)\right\|_{L^{\infty}}\left|X_{1}(s)-X_{2}(s)\right|+\left\|\left(E_{1}-E_{2}\right)(s)\right\|_{L^{\infty}} \\
& \leq C\left(\left|\left\|\left(E_{1}-E_{2}, A_{1}-A_{2}\right)(s)|\|+|\left(X_{1}-X_{2}\right)(s)|+|\left(P_{1}-P_{2}\right)(s) \mid\right) .\right.\right.
\end{aligned}
$$

Finally one gets from (3.42), (3.43), (3.44)

$$
\begin{aligned}
&\left|I_{1}(s)-I_{2}(s)\right| \leq C\left(1+\left|P_{1}(s)\right|+\left|P_{2}(s)\right|\right)\left(\left|\left\|\left|\left(E_{1}-E_{2}, A_{1}-A_{2}\right)(s)\right|\right\|\right.\right. \\
&\left.+\left|X_{1}(s)-X_{2}(s)\right|+\left|P_{1}(s)-P_{2}(s)\right|\right)\|\varphi\|_{L^{1}} \\
&+C\left(1+\left|P_{2}(s)\right|\right)\left|\int_{X_{2}(s) \mp(t-s)}^{X_{1}(s) \mp(t-s)} \varphi(u) d u\right| .
\end{aligned}
$$


Our conclusion follows from (3.36), (3.40), (3.41), (3.45).

Lemma 3.10. Assume that the hypotheses of Lemma 3.9 hold and $\int_{\mathbb{R}} \int_{\mathbb{R}}|p| f_{0} d p d x<$ $+\infty$. Then there is a constant $C$ depending on $T$ and the initial conditions such that for any function $\varphi \in C_{c}^{0}(\mathbb{R})$ and $\left(E_{k}, A_{k}\right) \in D_{T}, k \in\{1,2\}$, we have

$$
\begin{aligned}
& \left|\sum_{k=1}^{2}(-1)^{k} \int_{\mathbb{R}} \int_{\mathbb{R}} f_{0}(x, p) \int_{0}^{t} G\left(P_{k}(s), A_{k}\left(s, X_{k}(s)\right)\right) \frac{d}{d s}\left(\int_{0}^{X_{k}(s) \mp(t-s)} \varphi(u) d u\right) d s d p d x\right| \\
\leq & C\left(\left\|\left(A_{1}-A_{2}\right)(t)\right\|_{L^{\infty}}+\int_{0}^{t}\|\|\left(E_{1}-E_{2}, A_{1}-A_{2}\right)(s) \| d s\right)\|\varphi\|_{L^{1}} .
\end{aligned}
$$

Proof. By Lemma 3.4 we deduce that

$$
\begin{aligned}
\int_{\mathbb{R}} \int_{\mathbb{R}} f_{0}\left(1+\left|P_{1}(t)\right|+\left|P_{2}(t)\right|\right) d p d x & =\left\|f_{0}\right\|_{L^{1}}+\sum_{k=1}^{2} \int_{\mathbb{R}} \int_{\mathbb{R}} f_{E_{k}, A_{k}}(t, x, p)|p| d p d x \\
& \leq C \int_{\mathbb{R}} \int_{\mathbb{R}}(1+|p|) f_{0}(x, p) d p d x
\end{aligned}
$$

Performing similar computations as before (see also Remark 3.8) we obtain the estimate

$$
\begin{aligned}
\int_{\mathbb{R}} \int_{\mathbb{R}} f_{0}\left(1+\left|P_{2}(t)\right|\right)\left|\int_{X_{2}(t)}^{X_{1}(t)} \varphi(u) d u\right| d p d x & \leq C \int_{0}^{t}\left\|\mid\left(E_{1}-E_{2}, A_{1}-A_{2}\right)(s)\right\| \| d s \\
& \times\|\varphi\|_{L^{1}},
\end{aligned}
$$

and also

$$
\begin{gathered}
\int_{\mathbb{R}} \int_{\mathbb{R}} f_{0}\left(1+\left|P_{2}(s)\right|\right) \mid \int_{X_{2}(s) \mp(t-s)}^{X_{1}(s) \mp(t-s)} \varphi(u) d u d x \leq C \int_{0}^{s}\left\|\left(E_{1}-E_{2}, A_{1}-A_{2}\right)(\tau)\right\|\|d \tau\| \varphi \|_{L^{1}} \\
\leq C \int_{0}^{t}\left\|||\left(E_{1}-E_{2}, A_{1}-A_{2}\right)(\tau)\right\|\|d \tau\| \varphi \|_{L^{1}}, \forall s \in[0, t] .
\end{gathered}
$$

The conclusion follows by combining (3.35), (3.47), (3.48), (3.49) and using Proposition 2.3 .

We intend to apply Lemma 3.10 for estimating $Q_{6}=\int_{\mathbb{R}}\left(\partial_{x} \tilde{A}_{1}-\partial_{x} \tilde{A}_{2}\right) \varphi(x) d x=$ $-\frac{1}{2}\left(Q_{6}^{+}-Q_{6}^{-}\right)$(see (3.32) $)$. All we need to do is to check that the functions $G^{ \pm}$belong to the class $\mathcal{C}_{1}$. We have

$$
G^{ \pm}(P, A)=\frac{A}{P \pm \sqrt{1+|P|^{2}+|A|^{2}}}=\frac{A}{1+|A|^{2}}\left( \pm \sqrt{1+|P|^{2}+|A|^{2}}-P\right)
$$

and we check easily that

$$
\max \left\{\left|G^{ \pm}\right|,\left|\partial_{P} G^{ \pm}\right|,\left|\partial_{A} G^{ \pm}\right|\right\}(P, A) \leq g(A)(1+|P|), \forall(P, A) \in \mathbb{R}^{2},
$$

for some continuous function $g$. We have

$$
H^{ \pm}(P, A, B, C, E)=\left[C+\frac{P B}{\sqrt{1+|P|^{2}+|A|^{2}}}\right] \partial_{A} G^{ \pm}-\left[E+\frac{A B}{\sqrt{1+|P|^{2}+|A|^{2}}}\right] \partial_{P} G^{ \pm},
$$

and we check by direct computation that

$$
\max \left\{\left|H^{ \pm}\right|,\left|\partial_{P} H^{ \pm}\right|,\left|\partial_{A} H^{ \pm}\right|,\left|\partial_{B} H^{ \pm}\right|,\left|\partial_{C} H^{ \pm}\right|,\left|\partial_{E} H^{ \pm}\right|\right\} \leq h(A, B, C, E)(1+|P|),
$$


for some continuous function $h$. Therefore Lemma 3.10 applies, and by combining (3.32), (3.33) one gets for any function $\varphi \in C_{c}^{0}(\mathbb{R})$

$$
\begin{aligned}
\left|\int_{\mathbb{R}} \partial_{x}\left(\tilde{A}_{1}-\tilde{A}_{2}\right) \varphi(x) d x\right| & \leq C\left\|A_{1}(t)-A_{2}(t)\right\|_{L^{\infty}}\|\varphi\|_{L^{1}} \\
& +C \int_{0}^{t}\|\|\left(E_{1}-E_{2}, A_{1}-A_{2}\right)(s)\|\| d s\|\varphi\|_{L^{1}}
\end{aligned}
$$

Since we already know that $\partial_{x} \tilde{A}_{1}, \partial_{x} \tilde{A}_{2} \in L^{\infty}(\mathbb{R})$ we deduce by density that the previous inequality holds true for any function $\varphi \in L^{1}(\mathbb{R})$ and therefore we obtain

$$
\begin{aligned}
& \left\|\partial_{x} \tilde{A}_{1}(t)-\partial_{x} \tilde{A}_{2}(t)\right\|_{L^{\infty}} \\
& \quad \leq C\left\|A_{1}(t)-A_{2}(t)\right\|_{L^{\infty}} \\
& \quad+C \int_{0}^{t}\|\|\left(E_{1}-E_{2}, A_{1}-A_{2}\right)(s)\|\| d s, t \in[0, T] .
\end{aligned}
$$

Estimate for $\partial_{t} \tilde{A}_{1}-\partial_{t} \tilde{A}_{2}$

With the notations introduced in (3.32) we have for any function $\varphi \in L^{1}(\mathbb{R})$

$$
\int_{\mathbb{R}}\left(\partial_{t} \tilde{A}_{1}-\partial_{t} \tilde{A}_{2}\right) \varphi(x) d x=-\frac{1}{2} Q_{6}^{+}-\frac{1}{2} Q_{6}^{-},
$$

and therefore we obtain exactly as before that

$$
\begin{aligned}
\left\|\partial_{t} \tilde{A}_{1}(t)-\partial_{t} \tilde{A}_{2}(t)\right\|_{L^{\infty}} & \leq C\left\|A_{1}(t)-A_{2}(t)\right\|_{L^{\infty}} \\
& +C \int_{0}^{t}\|\|\left(E_{1}-E_{2}, A_{1}-A_{2}\right)(s)\|\| d s, \forall t \in[0, T] .
\end{aligned}
$$

Now collecting all the partial estimates of (3.25), (3.31), (3.51), (3.53) we deduce that there is a constant $C$ depending on $T$ and the initial conditions such that for any $\left(E_{k}, A_{k}\right) \in D_{T}, k \in\{1,2\}, t \in[0, T]$, we have

$$
\begin{aligned}
\left\|\left|\mathcal{F}\left(E_{1}, A_{1}\right)(t)-\mathcal{F}\left(E_{2}, A_{2}\right)(t)\right|\right\| & \leq C\left\|A_{1}(t)-A_{2}(t)\right\|_{L^{\infty}} \\
& +C \int_{0}^{t}\|\|\left(E_{1}-E_{2}, A_{1}-A_{2}\right)(s)\|\| d s .
\end{aligned}
$$

REMARK 3.11. A similar inequality holds in the QR case. We need to assume that $M_{2}=\int_{\mathbb{R}}|p|^{2} n_{0}(p) d p<+\infty, \int_{\mathbb{R}} \int_{\mathbb{R}}|p|^{2} f_{0} d p d x<+\infty$ and to work with the class $\mathcal{C}_{2}$.

4. Existence and uniqueness of a fixed point for $\mathcal{F}$. We start with a very easy lemma.

Lemma 4.1. Assume that $\alpha, \beta$ are nonnegative real numbers and $\left(z_{n}\right)_{n} \subset L^{\infty}(] 0, T[)$ is a sequence of nonnegative functions satisfying

$$
z_{n+2}(t) \leq \alpha \int_{0}^{t} z_{n}(s) d s+\beta \int_{0}^{t} z_{n+1}(s) d s, t \in[0, T], n \geq 0 .
$$

Then $\sum_{n \geq 0} z_{n}(t)$ converges uniformly on $[0, T]$ and we have

$$
\sum_{n \geq 0} z_{n}(t) \leq\left(\left\|z_{0}\right\|_{L^{\infty}}+\left\|z_{1}\right\|_{L^{\infty}}\right) \mathrm{e}^{(\alpha+\beta) t}, t \in[0, T] .
$$


Proof. Denote $S_{m}(t)=\sum_{n=0}^{m} z_{n}(t)$. By adding the inequalities (4.1) written for $n \in\{0,1,2, \ldots, m\}$ we obtain

$$
\begin{aligned}
S_{m+2}(t)-z_{0}(t)-z_{1}(t) & \leq \alpha \int_{0}^{t} S_{m}(s) d s+\beta \int_{0}^{t}\left(S_{m+1}(s)-z_{0}(s)\right) d s \\
& \leq(\alpha+\beta) \int_{0}^{t} S_{m+1}(s) d s,
\end{aligned}
$$

and therefore $S_{m+1}(t) \leq\left\|z_{0}\right\|_{L^{\infty}}+\left\|z_{1}\right\|_{L^{\infty}}+(\alpha+\beta) \int_{0}^{t} S_{m+1}(s) d s, \quad t \in[0, T], m \geq 0$. By Gronwall's lemma we deduce that $S_{m+1}(t) \leq\left(\left\|z_{0}\right\|_{L^{\infty}}+\left\|z_{1}\right\|_{L^{\infty}}\right) \mathrm{e}^{(\alpha+\beta) t}, t \in[0, T], m \geq$ 0 , and (4.2) follows by letting $m \rightarrow+\infty$. Combining (4.2) and the Lebesgue dominated convergence theorem yields $\lim _{n \rightarrow+\infty} \int_{0}^{T} z_{n}(s) d s=0$ and therefore, by the inequality (4.1) one gets the uniform convergence of $\left(z_{n}\right)_{n}$ towards 0 . As before, by adding the inequalities (4.1) written for $n, n+1, \ldots$ we obtain

$$
\sum_{k \geq n} z_{k}(t) \leq z_{n}(t)+z_{n+1}(t)+(\alpha+\beta) \int_{0}^{t} \sum_{k \geq n} z_{k}(s) d s .
$$

By Gronwall's lemma we have $\sum_{k \geq n} z_{k}(t) \leq\left(\left\|z_{n}\right\|_{L^{\infty}}+\left\|z_{n+1}\right\|_{L^{\infty}}\right) \mathrm{e}^{(\alpha+\beta) t}, t \in[0, T]$, $n \geq 0$, saying that $\sum_{n \geq 0} z_{n}$ converges uniformly on $[0, T]$.

By using successive approximations we prove the existence of a fixed point for $\mathcal{F}$ and we obtain the existence of solution for the system (1.12), (1.13), (1.14), (1.15), (1.16).

Theorem 4.2. Assume that $f_{0}$ in nonnegative, $(1+|p|) f_{0}$ belongs to $L^{1}\left(\mathbb{R}^{2}\right)$ and satisfies $(H),\left(H_{0}\right),\left(H_{1}\right),\left(H_{\infty}\right), \rho_{\text {ext }}$ is nonnegative, belongs to $L^{1}(\mathbb{R}) \cap L^{\infty}(\mathbb{R}), E_{0}^{\prime}=\rho_{\text {ext }}-$ $\int_{\mathbb{R}} f_{0} d p, A_{0} \in W^{2, \infty}(\mathbb{R})$, and $A_{1} \in W^{1, \infty}(\mathbb{R})$. Then for any $T>0$ there is at least one solution $(f, E, A)$ for the system (1.12), (1.13), (1.14), (1.15), (1.16) in the FR case, verifying $f \geq 0,(1+|p|) f \in L^{\infty}(] 0, T\left[; L^{1}\left(\mathbb{R}^{2}\right)\right), \int_{\mathbb{R}} f(\cdot, \cdot, p)(1+|p|) d p \in L^{\infty}(] 0, T[\times \mathbb{R})$, and $(E, A) \in D_{T}$.

Proof. We consider $\left(E_{0}, A_{0}\right)=(0,0) \in D_{T}$ and define $\left(E_{n}, A_{n}\right)=\mathcal{F}\left(E_{n-1}, A_{n-1}\right)$, for any $n \geq 1$ and $z_{n}(t)=\|\|\left(E_{n+1}-E_{n}, A_{n+1}-A_{n}\right)(t)\|\|, t \in[0, T], n \geq 0$. Observe that all functions $z_{n}$ are bounded on $[0, T]$ since $\left\|z_{n}\right\|_{L^{\infty}} \leq 2\left(e+a(T)+2 a_{1}(T)\right), \quad \forall n \geq 0$. The inequality (3.54) implies

$$
\begin{aligned}
z_{n+2}(t) & \leq C\left[\left\|A_{n+2}(t)-A_{n+1}(t)\right\|_{L^{\infty}}+\int_{0}^{t}\left\|\left(E_{n+2}, A_{n+2}\right)(s)-\left(E_{n+1}, A_{n+1}\right)(s)\right\| \| d s\right] \\
& =C\left(\left\|A_{n+2}(t)-A_{n+1}(t)\right\|_{L^{\infty}}+\int_{0}^{t} z_{n+1}(s) d s\right) .
\end{aligned}
$$

But (3.31) yields

$$
\left\|A_{n+2}(t)-A_{n+1}(t)\right\|_{L^{\infty}} \leq C \int_{0}^{t} \mid\left\|\left(E_{n+1}, A_{n+1}\right)(s)-\left(E_{n}, A_{n}\right)(s)\right\| \| d s=C \int_{0}^{t} z_{n}(s) d s .
$$

Finally one gets that there is a constant $C$ depending on $T$ and the initial conditions such that $z_{n+2}(t) \leq C \int_{0}^{t}\left(z_{n}(s)+z_{n+1}(s)\right) d s, \quad \forall t \in[0, T], n \geq 0$. Lemma 4.1 implies that $\left(E_{n}, A_{n}\right)_{n}$ is a Cauchy sequence in $Y_{T}=L^{\infty}(] 0, T[\times \mathbb{R}) \times W^{1, \infty}(] 0, T[\times \mathbb{R})$ and therefore converges to some fields $(E, A)$ in $Y_{T}$. Actually since $\left(E_{n}, A_{n}\right)_{n} \subset D_{T}$ we 
obtain immediately that $(E, A) \in D_{T}$. We check easily that $(E, A)$ is a fixed point of $\mathcal{F}$. We take $f=f_{E, A}$ and thus $(f, E, A)$ solve (1.12), (1.13). As in the proofs of Propositions 3.1. 3.6 we check that (1.15), (1.14) hold. The estimates for $\|(1+|p|) f\|_{\left.L^{\infty}(] 0, T ; L^{1}\left(\mathbb{R}^{2}\right)\right)}$ and $\left\|\int_{\mathbb{R}} f(\cdot, \cdot, p)(1+|p|) d p\right\|_{L^{\infty}(] 0, T[\times \mathbb{R})}$ follow from Lemmas 3.4, 3.5.

Theorem 4.3. Assume that the hypotheses of Theorem 4.2 are satisfied. Then there is at most one mild solution $(f, E, A)$ (i.e., $(E, A) \in L^{\infty}(] 0, T\left[; W^{1, \infty}(\mathbb{R})\right) \times L^{\infty}(] 0, T\left[; W^{2, \infty}(\mathbb{R})\right)$ and $f$ the solution by characteristics) for the system (1.12), (1.13), (1.14), (1.15), (1.16) in the FR case.

Proof. Suppose that $\left(f_{k}, E_{k}, A_{k}\right)_{k \in\{1,2\}}$ are two mild solutions. By computations similar to those in the proofs of Propositions 3.1, 3.2 (see also Propositions 3.6, 3.7) we show that $\left(E_{k}, A_{k}\right) \in D_{T}, k \in\{1,2\}$. Using (3.54) and (3.31) yields

$$
\left\|\left(E_{1}, A_{1}\right)(t)-\left(E_{2}, A_{2}\right)(t)\right\| \leq \leq \int_{0}^{t}\|\|\left(E_{1}, A_{1}\right)(s)-\left(E_{2}, A_{2}\right)(s)\|\| d s .
$$

The conclusion follows immediately by Gronwall's lemma.

Finally observe that the solution constructed above preserves the total energy. The proof is standard and is left to the reader.

Proposition 4.4. Assume that the hypotheses of Theorem 4.2 are satisfied and denote by $(f, E, A)$ the unique solution of the system (1.12), (1.13), (1.14), (1.15), (1.16). If the initial energy is finite i.e.,

$W_{0}:=\int_{\mathbb{R}} \int_{\mathbb{R}} f_{0} \sqrt{1+|p|^{2}+\left|A_{0}(x)\right|^{2}} d p d x+\frac{1}{2} \int_{\mathbb{R}}\left\{\left|E_{0}(x)\right|^{2}+\left|A_{0}^{\prime}(x)\right|^{2}+\left|A_{1}(x)\right|^{2}\right\} d x<+\infty$, then we have $W(t)=W_{0}$ for any $t \in[0, T]$ where

$$
W(t):=\int_{\mathbb{R}} \int_{\mathbb{R}} f \sqrt{1+|p|^{2}+|A(t, x)|^{2}} d p d x+\frac{1}{2} \int_{\mathbb{R}}\left\{|E(t, x)|^{2}+\left|\partial_{x} A\right|^{2}+\left|\partial_{t} A\right|^{2}\right\} d x .
$$

Acknowledgement. The author is thankful to J. A. Carrillo and S. Labrunie for helpful remarks and advice.

\section{REFERENCES}

[1] M. Bostan, Existence and uniqueness of the mild solution for the $1 D$ Vlasov-Poisson initialboundary value problem, SIAM J. Math. Anal. 37 (2005), 156-188. MR2176927

[2] F. Bouchut, F. Golse and C. Pallard, Classical solutions and the Glassey-Strauss theorem for the $3 D$ Vlasov-Maxwell system, Arch. Ration. Mech. Anal. 170 (2003), 1-15. MR 2012645|(2004i:82062)

[3] S. Calogero and G. Rein, On classical solutions of the Nordstrom-Vlasov system, Comm. Partial Differential Equations 28 (2003), 1863-1885. MR2015405 (2005c:35282)

[4] S. Calogero and G. Rein, Global weak solutions to the Nordstrom-Vlasov system, J. Differential Equations 204 (2004), 323-338. MR2085540 (2005h:83164)

[5] J.A. Carrillo and S. Labrunie, Global solutions for the one-dimensional Vlasov-Maxwell system for laser-plasma interaction, Math. Models Methods Appl. Sci. 16 (2006), 19-57. MR2194980

[6] J. Cooper and A. Klimas, Boundary-value problem for the Vlasov-Maxwell equation in one dimension, J. Math. Anal. Appl. 75 (1980), 306-329. MR581821 (81h:78004)

[7] R. J. Diperna and P.-L. Lions, Global weak solutions of the Vlasov-Maxwell system, Comm. Pure Appl. Math. XVII (1989), 729-757. MR1003433 (90i:35236)

[8] F. Filbet, Y. Guo and C.-W. Shu, Analysis of the relativistic Vlasov-Maxwell model in an interval, Quart. Appl. Math. 63 (2005), 691-714. MR2187927 (2006h:35267) 
[9] R. Glassey and J. Schaeffer, On the 'one and one-half dimensional' relativistic Vlasov-Maxwell system, Math. Methods Appl. Sci. 13 (1990), 169-179. MR1066384 (91g:82054)

[10] R.T. Glasey and J. Schaeffer, The two and one-half dimensional relativistic Vlasov-Maxwell system, Comm. Math. Phys. 185 (1997), 257-284. MR1463042 (98f:35143)

[11] R. Glassey and W. Strauss, Singularity formation in a collisionless plasma could only occur at high velocities, Arch. Ration. Mech. Anal. 92 (1986), 56-90. MR816621 (87j:82064)

[12] R. Glassey and W. Strauss, Large velocities in the relativistic Vlasov-Maxwell equations, J. Fac. Sci. Tokyo 36 (1989), 615-627. MR1039487 (91b:82059)

[13] Y. Guo, Global weak solutions of the Vlasov-Maxwell system with boundary conditions, Comm. Math. Phys. 154 (1993), 245-263. MR:1224079 (94c:35117)

[14] S. Klainerman and G. Staffilani, A new approach to study the Vlasov-Maxwell system, Comm. Pure Appl. Anal. 1 (2002), 103-125. MR1877669 (2003a:82065)

[15] F. Poupaud, Boundary value problems for the stationary Vlasov-Maxwell system, Forum Math. 4 (1992), 499-527. MR1176884 (93i:35144) 